Pure and Applied Mathematics Quarterly

Volume 3, Number 3

(Special Issue: In honor of

Leon Simon, Part 2 of 2$)$

$841-879,2007$

\title{
Measure-Theoretic Analysis and Nonlinear Conservation Laws
}

\author{
Gui-Qiang Chen Monica Torres William P. Ziemer \\ To Leon Simon with Friendship and Admiration
}

\begin{abstract}
We discuss some recent developments and trends of applying measure-theoretic analysis to the study of nonlinear conservation laws. We focus particularly on entropy solutions without bounded variation and Cauchy fluxes on oriented surfaces which are used to formulate the balance law. Our analysis employs the Gauss-Green formula and normal traces for divergence-measure fields, Young measures and compensated compactness, blow-up and scaling techniques, entropy methods, and related measuretheoretic techniques. Nonlinear conservation laws include multidimensional scalar conservation laws, strictly hyperbolic systems of conservation laws, isentropic Euler equations, two-dimensional sonic-subsonic flows, and degenerate parabolic-hyperbolic equations. Some open problems and trends on the topics are addressed and an extensive list of references is also provided.

Key Words and Phrases. Measure-theoretic analysis, geometric measures, conservation laws, Cauchy fluxes, balance laws, entropy solutions, divergence-measure fields, sets of finite perimeter, Gauss-Green formula, normal traces, oriented surfaces, compactness, Young measures, compensated compactness, blow-up, scaling, axioms for continuum thermodynamics, field equations.
\end{abstract}

Received July 2, 2006.

1991 Mathematics Subject Classification. Primary: 28C05, 35L65,35L50; Secondary: 26B12, $28 \mathrm{~A} 75$. 


\section{INTRODUCTION}

We are concerned with entropy solutions to nonlinear conservation laws:

$$
\partial_{t} \mathbf{u}+\nabla_{\mathbf{x}} \cdot \mathbf{f}(\mathbf{u})=0, \quad \mathbf{u} \in \mathbb{R}^{m}, \quad \mathbf{x} \in \mathbb{R}^{d},
$$

where $\nabla_{\mathbf{x}}=\left(\partial_{x_{1}}, \ldots, \partial_{x_{d}}\right)$, and

$$
\mathbf{f}=\left(\mathbf{f}_{1}, \ldots, \mathbf{f}_{d}\right): \mathbb{R}^{m} \rightarrow\left(\mathbb{R}^{m}\right)^{d}
$$

is a nonlinear mapping with $\mathbf{f}_{i}: \mathbb{R}^{m} \rightarrow \mathbb{R}^{m}$ for $i=1, \ldots, d$.

One of the main difficulties in dealing with (1.1) is that solutions of the Cauchy problem (1.1) with Cauchy data,

$$
\left.\mathbf{u}\right|_{t=0}=\mathbf{u}_{0}
$$

generally develop singularities in finite time (even when starting out from smooth initial data), because, in part, of the physical phenomena of focusing and breaking of waves and the development of shock waves and vortices. For this reason, one is forced to consider solutions in the space of discontinuous functions. Therefore, one can not directly use many of the classical, powerful analytic techniques that predominate in the theory of partial differential equations.

For one-dimensional strictly hyperbolic systems, Glimm's theorem [54] indicates that, as long as $\left\|\mathbf{u}_{0}\right\|_{B V}$ is sufficiently small, the solution $\mathbf{u}(t, x), x \in \mathbb{R}$, satisfies the following stability estimate:

$$
\|\mathbf{u}(t, \cdot)\|_{B V} \leq C\left\|\mathbf{u}_{0}\right\|_{B V} .
$$

Even more, for two solutions $\mathbf{u}(t, x)$ and $\mathbf{v}(t, x)$ with small total variation obtained by the Glimm scheme, the wave-front tracking method, or the vanishing viscosity method,

$$
\|\mathbf{u}(t, \cdot)-\mathbf{v}(t, \cdot)\|_{L^{1}(\mathbb{R})} \leq C\|\mathbf{u}(0, \cdot)-\mathbf{v}(0, \cdot)\|_{L^{1}(\mathbb{R})} .
$$

For more details, see $[8,10,37,62,67]$ and the references cited therein.

The recent great progress on the one-dimensional theory of hyperbolic conservation laws based on $B V$ estimates and trace theorems of $B V$ fields raises the hope that a similar approach may also be effective for multidimensional hyperbolic systems of conservation laws; that is, whether entropy solutions satisfy the relatively modest stability estimate:

$$
\|\mathbf{u}(t, \cdot)\|_{B V} \leq C\left\|\mathbf{u}_{0}\right\|_{B V} .
$$

Unfortunately, this is not the case. It was shown by Rauch [88] that a necessary condition for (1.4) is

$$
\nabla \mathbf{f}_{k}(\mathbf{u}) \nabla \mathbf{f}_{l}(\mathbf{u})=\nabla \mathbf{f}_{l}(\mathbf{u}) \nabla \mathbf{f}_{k}(\mathbf{u}) \quad \text { for all } k, l=1,2, \ldots, d .
$$

This suggests that, if one wishes to frame the problem within $B V$, then it is necessary to consider only systems in which the commutation relation (1.5) holds. 
This special case includes the scalar case $m=1$ and the one-dimensional case $d=1$. Beyond that, it contains very few systems of physical interest. One example is the Keyfitz-Kranzer system with flux functions:

$$
\mathbf{f}_{k}(\mathbf{u})=\phi_{k}\left(|\mathbf{u}|^{2}\right) \mathbf{u}, \quad k=1,2, \ldots, d,
$$

which governs the flow of a fluid in an anisotropic porous medium. However, the recent study in Ambrosio-De Lellis [3] and Bressan [11] shows that, even in this case, (1.4) fails. De Lellis in [39] further showed that, for system (1.6), the $B V$-norm of solutions can even blow up in finite time. Thus, the space $B V$ is not a well-posed space.

Moreover, even for a one-dimensional system whose strict hyperbolicity fails or whose initial data is allowed to be of large amplitude, the solution is, in general, no longer in $B V$. However, some bounds in $L^{\infty}$ or $L^{p}$ may be achieved. One of such examples is the isentropic Euler equations, for which we have

$$
\|\mathbf{u}(t, \cdot)\|_{L^{\infty}} \leq C\left\|\mathbf{u}_{0}\right\|_{L^{\infty}} ;
$$

see Chen-LeFloch [25] and the references cited therein. Furthermore, for the multidimensional case, entropy solutions generally do not even have the relatively modest stability:

$$
\|\mathbf{u}(t, \cdot)-\overline{\mathbf{u}}\|_{L^{p}} \leq C_{p}\left\|\mathbf{u}_{0}-\overline{\mathbf{u}}_{0}\right\|_{L^{p}}, \quad p \neq 2,
$$

for some constant state $\bar{u}$ (also see Brenner [9] for the linear case).

Naturally, we want to treat the questions of existence, stability, uniqueness, compactness, and long-time behavior of entropy solutions for multidimensional conservation laws with as much generality as possible. In this regard, it is important to develop a suitable measure-theoretic analysis and identify a good analytic framework for studying entropy solutions of (1.1), which are not in $B V$ (or even in $L^{p}$ ). The most general framework is the space of divergence-measure fields, formulated recently in Chen-Frid [22] (also see Chen-Torres [31] and Chen-TorresZiemer [32]), which is based on the connection between divergence-measure fields and entropy solutions to conservation laws. In Section 2, we exhibit a natural connection between entropy solutions of (1.1) and divergence-measure fields. We then proceed to develop a theory for these fields and show how they may serve to develop an analytic framework for studying entropy solutions of (1.1) via measure-theoretic analysis.

In Section 3, we are concerned with the compactness of exact or approximate solutions without bounded variation for (1.1). For this, we discuss a fundamental representation theorem for weak limits via Young measures and the theory of compensated compactness. In Section 4, we discuss a connection between Cauchy fluxes and divergence-measure fields and exhibit a general framework which is fundamental for the derivation of nonlinear systems of balance laws with measure-valued source terms from the physical principle of balance law. Then, 
in Section 5, we discuss some recent results and developments, by using measuretheoretic analysis, for entropy solutions in $L^{\infty}$ for multidimensional scalar conservation laws. In Section 6, we present applications of measure-theoretic analysis to systems of conservation laws.

Finally, we address some open problems and trends in analyzing entropy solutions without bounded variation. We also provide an extensive list of references for further ideas, results, and approaches on measure-theoretic analysis and nonlinear conservation laws.

\section{Divergence-Measure Fields and Nonlinear Conservation Laws}

In this section we first discuss a natural connection between entropy solutions of (1.1) and a class of vector fields, called divergence-measure fields, and exhibit a theory for these fields as a basis to develop analytic frameworks and approaches for studying entropy solutions of (1.1) via measure-theoretic analysis. We denote $\mathbb{R}_{+}^{d+1}:=\mathbb{R}_{+} \times \mathbb{R}^{d}=(0, \infty) \times \mathbb{R}^{d}$ throughout this paper.

Consider the following class of entropy solutions $\mathbf{u}(t, \mathbf{x})$ to $(1.1)$ :

(i) $\mathbf{u}(t, \mathbf{x}) \in \mathcal{M}\left(\mathbb{R}_{+}^{d+1}\right)$ or $\quad L^{p}\left(\mathbb{R}_{+}^{d+1}\right), 1 \leq p \leq \infty$;

(ii) For any convex entropy-entropy flux pair $(\eta, \mathbf{q})$ so that $(\eta(\mathbf{u}), \mathbf{q}(\mathbf{u}))(t, \mathbf{x})$ is a distributional field,

$$
\mu_{\eta}:=\partial_{t} \eta(\mathbf{u})+\nabla_{\mathbf{x}} \cdot \mathbf{q}(\mathbf{u}) \leq 0
$$

in the sense of distributions, where an entropy-entropy flux pair $(\eta, \mathbf{q}):=$ $\left(\eta, q_{1}, \ldots, q_{d}\right)$ (as a solution of $\left.\nabla q_{k}(\mathbf{u})=\nabla \eta(\mathbf{u}) \nabla \mathbf{f}_{k}(\mathbf{u}), k=1,2, \ldots, d\right)$ is simply called an entropy pair later.

Clearly, any entropy solution is a weak solution as can be seen by choosing $\eta(\mathbf{u})= \pm \mathbf{u}$ in $(2.1)$.

One of the main issues in conservation laws is to study the behavior of entropy solutions in this class to explore to the fullest extent possible all questions relating to large-time behavior, uniqueness, stability, structure, and traces of entropy solutions, with neither specific reference to any particular method for constructing the solutions nor additional regularity assumptions.

From (2.1), the Schwartz Lemma implies that the distribution $\mu_{\eta}$ is in fact a Radon measure:

$$
\mu_{\eta}=\operatorname{div}_{(t, \mathbf{x})}(\eta(\mathbf{u}(t, \mathbf{x})), \mathbf{q}(\mathbf{u}(t, \mathbf{x}))) \in \mathcal{M}\left(\mathbb{R}_{+}^{d+1}\right) .
$$


Furthermore, when $\mathbf{u} \in L^{\infty}$, this is also true for any $C^{2}$ entropy pair $(\eta, \mathbf{q})(\eta$ not necessarily convex) if (1.1) has a strictly convex entropy, as first observed by Chen [17]. More generally, we have

Definition 2.1. Let $\mathbb{D} \subset \mathbb{R}^{N}$ be open. For $1 \leq p \leq \infty, \mathbf{F}$ is called a $\mathcal{D} \mathcal{M}^{p}(\mathbb{D})$ field if $\mathbf{F} \in L^{p}(\mathbb{D})$ and

$$
\|\mathbf{F}\|_{\mathcal{D M}^{p}(\mathbb{D})}:=\|\mathbf{F}\|_{L^{p}\left(\mathbb{D} ; \mathbb{R}^{N}\right)}+\|\operatorname{div} \mathbf{F}\|_{\mathcal{M}(\mathbb{D})}<\infty ;
$$

and the field $\mathbf{F}$ is called a $\mathcal{D} \mathcal{M}^{\text {ext }}(\mathbb{D})$-field if $\mathbf{F} \in \mathcal{M}(\mathbb{D})$ and

$$
\|\mathbf{F}\|_{\mathcal{D M}^{e x t}(\mathbb{D})}:=\|(\mathbf{F}, \operatorname{div} \mathbf{F})\|_{\mathcal{M}(\mathbb{D})}<\infty .
$$

Furthermore, $\mathbf{F}$ is called a $\mathcal{D} \mathcal{M}_{l o c}^{p}\left(\mathbb{R}^{N}\right)$ field if $\mathbf{F} \in \mathcal{D} \mathcal{M}^{p}(\mathbb{D})$ and $\mathbf{F}$ called a $\mathcal{D} \mathcal{M}_{\text {loc }}^{\text {ext }}\left(\mathbb{R}^{N}\right)$ if $\mathbf{F} \in \mathcal{D} \mathcal{M}^{\text {ext }}(\mathbb{D})$, for any bounded open set $\mathbb{D} \subset \mathbb{R}^{N}$. A field $\mathbf{F}$ is simply called a $\mathcal{D} \mathcal{M}$-field in $\mathbb{D}$ if $\mathbf{F} \in \mathcal{D} \mathcal{M}^{p}(\mathbb{D}), 1 \leq p \leq \infty$, or $\mathbf{F} \in \mathcal{D M}^{\text {ext }}(\mathbb{D})$.

These spaces, under the respective norms $\|\mathbf{F}\|_{\mathcal{D M}^{p}(\mathbb{D})}$ and $\|\mathbf{F}\|_{\mathcal{D M}^{e x t}(\mathbb{D})}$, are Banach spaces, which are larger than the space of $B V$ fields. $B V$ theory (cf. Federer [48] and Volpert-Hudjaev [105]; also Ambrosio-Fusco-Pallara [2], Burago-Maźya [13], and Ziemer [112]), especially the Gauss-Green formula and traces of $B V$ functions, has significantly advanced our understanding of solutions of nonlinear partial differential equations and related problems in the calculus of variations, differential geometry, and other areas; as an example, the one-dimensional theory of hyperbolic conservation laws. A natural question is to what extent the $\mathcal{D M}$-fields, along with the development of normal traces and the Gauss-Green theorem, can deal with entropy solutions for multidimensional conservation laws. At a first glance, it seems impossible because of the following example by Whitney in 1957.

EXAmple 2.1 (Whitney [107]): The field

$$
\mathbf{F}\left(y_{1}, y_{2}\right)=\left(\frac{-y_{2}}{y_{1}^{2}+y_{2}^{2}}, \frac{y_{1}}{y_{1}^{2}+y_{2}^{2}}\right)
$$

belongs to $\mathcal{D} \mathcal{M}_{\text {loc }}^{1}\left(\mathbb{R}^{2}\right)$. However, for $\Omega=\left\{\mathbf{y}:|\mathbf{y}|<1, y_{2}>0\right\}$,

$$
\int_{\Omega} \operatorname{div} \mathbf{F}=0 \neq-\int_{\partial \Omega} \mathbf{F} \cdot \nu d \mathcal{H}^{1}=\pi,
$$

if one understands $\mathbf{F} \cdot \nu$ in the classical sense, where $\nu$ is the interior unit normal on $\partial \Omega$ to $\Omega$. This implies that, in general, the classical Gauss-Green theorem fails for a $\mathcal{D} \mathcal{M}$-field.

ExAMPLE 2.2: For any $\mu_{i} \in \mathcal{M}(\mathbb{R}), i=1,2$, with finite total variation,

$$
\mathbf{F}\left(y_{1}, y_{2}\right)=\left(\mu_{1}\left(y_{2}\right), \mu_{2}\left(y_{1}\right)\right) \in \mathcal{D} \mathcal{M}^{e x t}\left(\mathbb{R}^{2}\right) .
$$


A non-trivial example of such fields is provided by the Riemann solutions of the one-dimensional Euler equations in Lagrangian coordinates for which the vacuum generally develops (see Chen-Frid [22]).

Some earlier efforts were made on generalizing the Gauss-Green theorem for some special situations, and relevant results can be found in Anzellotti [4] for an abstract formulation for $\mathbf{F} \in L^{\infty}$ over domains with $C^{1}$ boundary, and Ziemer [111] for a related problem for $\operatorname{div} \mathbf{F} \in L^{\infty}$ over sets of finite perimeter; also see $[1,5,12,90,112]$. In Chen-Frid [22], it was shown that a generalized Gauss-Green formula holds for $\mathbf{F} \in \mathcal{D} \mathcal{M}^{\infty}$, by demonstrating a way to explicitly calculate the suitable normal traces over Lipschitz deformable domains. This motivated the development of a theory of divergence-measure fields in [22, 31, 32] and related applications (cf. [22, 23, 25, 30, 40, 65, 104]).

We now discuss the Gauss-Green formula for $\mathcal{D} \mathcal{M}$-fields over $\Omega \subset \mathbb{D}$ by introducing a suitable notion of normal traces over the boundary $\partial \Omega$ of a bounded open set with Lipschitz deformable boundary, established in Chen-Frid [22].

Definition 2.2. Let $\Omega \subset \mathbb{R}^{N}$ be an open bounded subset. We say that $\partial \Omega$ is a deformable Lipschitz boundary, provided that

(i) For any $\mathbf{x} \in \partial \Omega$, there exist $r>0$ and a Lipschitz map $\gamma: \mathbb{R}^{N-1} \rightarrow \mathbb{R}$ such that, after rotating and relabeling coordinates if necessary,

$$
\Omega \cap Q(\mathbf{x}, r)=\left\{\mathbf{y} \in \mathbb{R}^{N}: \gamma\left(y_{1}, \ldots, y_{N-1}\right)<y_{N}\right\} \cap Q(\mathbf{x}, r),
$$

where $Q(\mathbf{x}, r)=\left\{\mathbf{y} \in \mathbb{R}^{N}:\left|y_{i}-x_{i}\right| \leq r, i=1, \ldots, N\right\}$;

(ii) There exists $\Psi: \partial \Omega \times[0,1] \rightarrow \bar{\Omega}$ such that $\Psi$ is a homeomorphism, biLipschitz over its image, and $\Psi(\omega, 0)=\omega$ for any $\omega \in \partial \Omega$. The map $\Psi$ is called a Lipschitz deformation of the boundary $\partial \Omega$.

Denote $\partial \Omega_{s}:=\Psi(\partial \Omega \times\{s\}), s \in[0,1]$, and denote by $\Omega_{s}$ the open subset of $\Omega$ bounded by $\partial \Omega_{s}$.

Remark 2.1. The domains with deformable Lipschitz boundaries clearly include star-shaped domains and domains whose boundaries satisfy the cone property. It is also clear that, if $\Omega$ is the image through a bi-Lipschitz map of a domain $\bar{\Omega}$ with a Lipschitz deformable boundary, then $\Omega$ itself possesses a Lipschitz deformable boundary.

For $\mathcal{D M}^{p}$-fields with $1<p \leq \infty$, we have

Theorem 2.1 (Chen-Frid [22]). Let $\mathbf{F} \in \mathcal{D M}^{p}(\mathbb{D}), 1<p \leq \infty$. Let $\Omega \subset \mathbb{D}$ be a bounded open set with Lipschitz deformable boundary. Then there exists a 
continuous linear functional $\mathbf{F} \cdot \nu$ over $\operatorname{Lip}(\partial \Omega)$ such that, for any $\phi \in \operatorname{Lip}\left(\mathbb{R}^{N}\right)$,

$$
\langle\mathbf{F} \cdot \nu, \phi\rangle_{\partial \Omega}=-\langle\operatorname{div} \mathbf{F}, \phi\rangle_{\Omega}-\int_{\Omega} \nabla \phi \cdot \mathbf{F} d \mathbf{y} .
$$

Moreover, let $h: \mathbb{R}^{N} \rightarrow \mathbb{R}$ be the level set function of $\partial \Omega_{s}$ :

$$
h(\mathbf{y}):= \begin{cases}0 & \text { for } \mathbf{y} \in \mathbb{R}^{N}-\bar{\Omega}, \\ 1 & \text { for } \mathbf{y} \in \Omega-\Psi(\partial \Omega \times[0,1]), \\ s & \text { for } \mathbf{y} \in \partial \Omega_{s}, 0 \leq s \leq 1 .\end{cases}
$$

Then, for any $\psi \in \operatorname{Lip}(\partial \Omega)$,

$$
\langle\mathbf{F} \cdot \nu, \psi\rangle_{\partial \Omega}=\lim _{s \rightarrow 0} \frac{1}{s} \int_{\Psi(\partial \Omega \times(0, s))} \mathcal{E}(\psi) \nabla h \cdot \mathbf{F} d \mathbf{y},
$$

where $\mathcal{E}(\psi)$ is any Lipschitz extension of $\psi$ to the whole space $\mathbb{R}^{N}$.

In the case $p=\infty$, the normal trace $\mathbf{F} \cdot \nu$ is a function in $L^{\infty}(\partial \Omega)$ satisfying

$$
\|\mathbf{F} \cdot \nu\|_{L^{\infty}(\partial \Omega)} \leq\|\mathbf{F}\|_{L^{\infty}(\Omega)}:=\left\|\sqrt{F_{1}^{2}+\cdots+F_{N}^{2}}\right\|_{L^{\infty}(\Omega)} .
$$

Furthermore, for any field $\mathbf{F} \in \mathcal{D} \mathcal{M}^{\infty}(\mathbb{D})$,

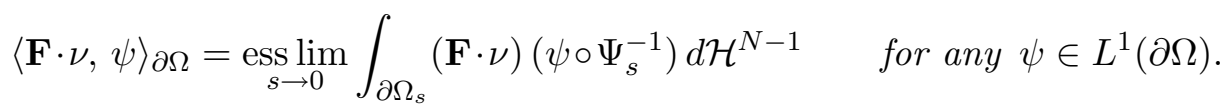

Finally, for $\mathbf{F} \in \mathcal{D} \mathcal{M}^{p}(\mathbb{D})$ with $1<p<\infty, \mathbf{F} \cdot \nu$ can be extended to a continuous linear functional over $W^{1-1 / p, p} \cap L^{\infty}(\partial \Omega)$.

EXAMPLE 2.3: The field

$$
\mathbf{F}\left(y_{1}, y_{2}\right)=\left(\sin \left(\frac{1}{y_{1}-y_{2}}\right), \sin \left(\frac{1}{y_{1}-y_{2}}\right)\right)
$$

belongs to $\mathcal{D} \mathcal{M}^{\infty}\left(\mathbb{R}^{2}\right)$. It is impossible to define any reasonable notion of traces over the line $y_{1}=y_{2}$ for the component $\sin \left(\frac{1}{y_{1}-y_{2}}\right)$. Nevertheless, the unit normal $\nu_{\tau}$ to the line $y_{1}-y_{2}=\tau$ is the vector $\left(\frac{1}{\sqrt{2}},-\frac{1}{\sqrt{2}}\right)$ so that the scalar product $\mathbf{F}\left(y_{1}, y_{1}-\tau\right) \cdot \nu_{\tau}$ is identically zero over this line. Hence, we find that

$$
\mathbf{F} \cdot \nu \equiv 0 \quad \text { over the line } y_{1}=y_{2}
$$

and the Gauss-Green formula implies that, for any $\phi \in C_{0}^{1}\left(\mathbb{R}^{2}\right)$,

$$
0=\left\langle\left.\operatorname{div} \mathbf{F}\right|_{y_{1}>y_{2}}, \phi\right\rangle=-\int_{y_{1}>y_{2}} \mathbf{F} \cdot \nabla \phi d \mathbf{y} .
$$

This identity may be directly obtained by applying the Dominated Convergence Theorem to the analogous identity from the classical Gauss-Green formula.

As indicated by Examples 2.1-2.2, there is a subtlety involved with defining the normal trace for fields in $\mathcal{D} \mathcal{M}^{1}$ and $\mathcal{D} \mathcal{M}^{\text {ext }}$. Thus, we have to define the 
normal trace as a functional over the $\operatorname{spaces} \operatorname{Lip}(\gamma, \partial \Omega)$ with $\gamma>1$ (see Stein [99], Chapter 6).

For $1<\gamma \leq 2$, the elements of $\operatorname{Lip}(\gamma, \partial \Omega)$ are vectors with $(N+1)$-components, where the first component is the function itself and the other $N$ components are its "first-order partial derivatives". In particular, as a functional over $\operatorname{Lip}(\gamma, \partial \Omega)$, the values of the normal trace of a field in $\mathcal{D} \mathcal{M}^{1}$ or $\mathcal{D} \mathcal{M}^{\text {ext }}$ on $\partial \Omega$ depend on not only the values of the respective functions over $\partial \Omega$, but also the values of their first-order derivatives over $\partial \Omega$. To define the normal traces for $\mathbf{F} \in \mathcal{D} \mathcal{M}^{1}(\Omega)$ or $\mathcal{D} \mathcal{M}^{\text {ext }}(\Omega)$, we resort to the properties of the Whitney extensions of functions in $\operatorname{Lip}(\gamma, \partial \Omega)$ to $\operatorname{Lip}\left(\gamma, \mathbb{R}^{N}\right)$.

We have the following analogue of Theorem 2.1 which involves vector fields in $\mathcal{D} \mathcal{M}^{1}$ and $\mathcal{D} \mathcal{M}^{\text {ext }}$.

Theorem 2.2 (Chen-Frid [23]). Let $\mathbf{F} \in \mathcal{D} \mathcal{M}^{1}(\mathbb{D})$ or $\mathcal{D} \mathcal{M}^{\text {ext }}(\mathbb{D})$. Let $\Omega \subset \mathbb{D}$ be a bounded open set with Lipschitz deformable boundary. Then there exists a continuous linear functional $\mathbf{F} \cdot \nu$ over $\operatorname{Lip}(\gamma, \partial \Omega)$ for any $\gamma>1$ such that, for any $\phi \in \operatorname{Lip}\left(\gamma, \mathbb{R}^{N}\right)$,

$$
\langle\mathbf{F} \cdot \nu, \phi\rangle_{\partial \Omega}=-\langle\operatorname{div} \mathbf{F}, \phi\rangle_{\Omega}-\langle\mathbf{F}, \nabla \phi\rangle_{\Omega}
$$

Moreover, let $h: \mathbb{R}^{N} \rightarrow \mathbb{R}$ be the level set function as defined in Theorem 2.1; and, in the case that $\mathbf{F} \in \mathcal{D} \mathcal{M}^{\text {ext }}(\mathbb{D})$, assume also that $\partial_{x_{i}} h$ is $\left|F_{i}\right|$-measurable and its set of non-Lebesgue points has $\left|F_{i}\right|$-measure zero, $i=1, \ldots, N$. Then, for any $\psi \in \operatorname{Lip}(\gamma, \partial \Omega), \gamma>1$,

$$
\langle\mathbf{F} \cdot \nu, \psi\rangle_{\partial \Omega}=\lim _{s \rightarrow 0} \frac{1}{s}\langle\mathbf{F}, \mathcal{E}(\psi) \nabla h\rangle_{\Psi(\partial \Omega \times(0, s))},
$$

where $\mathcal{E}(\psi) \in \operatorname{Lip}\left(\gamma, \mathbb{R}^{N}\right)$ is the Whitney extension of $\psi$ on $\partial \Omega$ to $\mathbb{R}^{N}$.

Remark 2.2. For $\mathbf{F} \in \mathcal{D} \mathcal{M}^{1}(\mathbb{D})$ or $\mathcal{D} \mathcal{M}^{\text {ext }}(\mathbb{D})$, the normal trace $\mathbf{F} \cdot \nu$ may no longer be a function on $\partial \Omega$ in general; that is, it cannot be represented as an integrable function with respect to the $(n-1)$-dimensional Hausdorff measure over $\partial \Omega$. This can be seen in Example 2.1 for $\mathbf{F} \in \mathcal{D} \mathcal{M}_{l o c}^{1}\left(\mathbb{R}^{2}\right)$, for which $\mathbf{F} \cdot \nu$ is a measure over $\partial \Omega$.

The analysis over sets with Lipschitz boundary has been extended to the analysis over sets of finite perimeter for $\mathbf{F} \in \mathcal{D} \mathcal{M}^{\infty}(\mathbb{D})$.

Theorem 2.3 (Chen-Torres [31]). Let $\mathbf{F} \in \mathcal{D} \mathcal{M}^{\infty}(\mathbb{D})$. If $\Omega \Subset \mathbb{D}$ is a bounded set of finite perimeter, there exists an $\mathcal{H}^{N-1}$-integrable function (denoted by) $\mathbf{F} \cdot \nu \in L^{\infty}\left(\partial^{*} \Omega ; \mathcal{H}^{N-1}\right)$ such that, for any $\phi \in C_{0}^{1}\left(\mathbb{R}^{N}\right)$,

$$
\langle\mathbf{F} \cdot \nu, \phi\rangle_{\partial^{*} \Omega}=-\langle\operatorname{div} \mathbf{F}, \phi\rangle_{\Omega^{1}}-\langle\mathbf{F}, \nabla \phi\rangle_{\Omega^{1}},
$$

where $\partial^{*} \Omega$ denotes the measure-theoretic reduced boundary of $\Omega$ and $\Omega^{1}$ the measuretheoretic interior of $\Omega$. 
When $\partial \Omega$ is Lipschitz deformable, the normal trace introduced by Chen-Torres [31], coincides with the normal trace by Chen-Frid [22]. Even when $\Omega$ is a bounded set of finite perimeter, we have the following theorem.

Theorem 2.4 (Chen-Torres-Ziemer [32]). Let $\mathbf{F} \in \mathcal{D} \mathcal{M}^{\infty}(\mathbb{D})$. Let $\Omega:=\Omega^{1} \cup$ $\partial^{*} \Omega \Subset \mathbb{D}$ be a bounded set of finite perimeter. Then

(i) For almost every $s \in(1 / 2,1)$, there exists a signed measure $\sigma_{i}$ and a family of sets $A_{k ; s}$ with smooth boundaries such that

(a) $\|\operatorname{div} \mathbf{F}\|\left(\left(A_{k ; s} \backslash \Omega^{1}\right) \cup\left(\Omega^{1} \backslash A_{k ; s}\right)\right) \rightarrow 0$;

(b) $\lim _{k \rightarrow \infty} \mathcal{H}^{N-1}\left(\partial A_{k ; s} \cap\left(\Omega^{0} \cup \partial^{*} \Omega\right)\right)=0$, where $\Omega^{0}$ is the measure-theoretic exterior of $\Omega$;

(c) $\lim _{k \rightarrow \infty}\left\|\sigma_{k ; s}\right\|\left(\Omega^{0} \cup \partial^{*} \Omega\right)=0$, where the measures $\sigma_{k ; s}$ are defined by

$$
\sigma_{k ; s}(B)=\int_{B \cap \partial A_{k ; s}} \mathbf{F} \cdot \nu d \mathcal{H}^{N-1}
$$

for any Borel set $B \subset \mathbb{D}$ with $\mathbf{F} \cdot \nu$ being the normal trace over the smooth boundary $\partial A_{k ; s}$;

(d) $\sigma_{i}$ is carried by $\partial^{*} \Omega$ in the sense that $\left\|\sigma_{i}\right\|\left(\mathbb{D} \backslash \partial^{*} \Omega\right)=0$, where $\sigma_{i}:=$ $w^{*}-\lim _{k \rightarrow \infty} \sigma_{k ; s}$ in the measure sense which is independent of $s \in$ $(1 / 2,1)$;

(e) $\left\|\sigma_{i}\right\|<<\mathcal{H}^{N-1}\left\llcorner\partial^{*} \Omega\right.$;

(f) The density of $\sigma_{i},(\mathbf{F} \cdot \nu)_{i}$, is called the interior normal trace relative to $\Omega$ of $\mathbf{F}$ on $\partial^{*} \Omega$ and satisfies

$$
\int_{\Omega^{1}} \operatorname{div} \mathbf{F}=(\operatorname{div} \mathbf{F})\left(\Omega^{1}\right)=-\sigma_{i}\left(\partial^{*} \Omega\right)=-\int_{\partial^{*} \Omega}(\mathbf{F} \cdot \nu)_{i}(\mathbf{y}) d \mathcal{H}^{N-1}(\mathbf{y}) ;
$$

(g) $\left\|\sigma_{i}\right\|=\left\|(\mathbf{F} \cdot \nu)_{i}\right\|_{L^{\infty}\left(\partial^{*} \Omega, \mathcal{H}^{N-1}\right)} \leq\|\mathbf{F}\|_{L^{\infty}(\mathbb{D})}$.

(ii) For almost every $s \in(0,1 / 2)$, there exists a signed measure $\sigma_{e}$ and a family of sets $A_{k ; s}$ with smooth boundaries such that

(a) $\|\operatorname{div} \mathbf{F}\|\left(\left(A_{k ; s} \backslash \Omega^{1}\right) \cup\left(\Omega^{1} \backslash A_{k ; s}\right)\right) \rightarrow 0$;

(b) $\lim _{k \rightarrow \infty} \mathcal{H}^{N-1}\left(\partial A_{k ; s} \cap \Omega\right)=0$;

(c) $\lim _{k \rightarrow \infty}\left\|\sigma_{k ; s}\right\|\left(\mathbb{D} \backslash \Omega^{0}\right)=\lim _{k \rightarrow \infty}\left\|\sigma_{k ; s}\right\|(\Omega)=0$;

(d) $\sigma_{e}$ is carried by $\partial^{*} \Omega$ in the sense that $\left\|\sigma_{e}\right\|\left(\mathbb{D} \backslash \partial^{*} \Omega\right)=0$, where $\sigma_{e}:=$ $w^{*}-\lim _{k \rightarrow \infty} \sigma_{k ; s}$ in the measure sense which is independent of $s \in$ $(0,1 / 2)$;

(e) $\left\|\sigma_{e}\right\|<<\mathcal{H}^{N-1}\left\llcorner\partial^{*} \Omega\right.$;

(f) The density of $\sigma_{e},(\mathbf{F} \cdot \nu)_{e}$, is called the exterior normal trace relative to $\Omega$ of $\mathbf{F}$ on $\partial^{*} \Omega$ and satisfies

$$
\int_{\Omega} \operatorname{div} \mathbf{F}=(\operatorname{div} \mathbf{F})(\Omega)=-\sigma_{e}\left(\partial^{*} \Omega\right)=-\int_{\partial^{*} \Omega}(\mathbf{F} \cdot \nu)_{e}(\mathbf{y}) d \mathcal{H}^{N-1}(\mathbf{y}) ;
$$


(g) $\left\|\sigma_{e}\right\|=\left\|(\mathbf{F} \cdot \nu)_{e}\right\|_{L^{\infty}\left(\partial^{*} \Omega ; \mathcal{H}^{N-1}\right)} \leq\|\mathbf{F}\|_{L^{\infty}(\mathbb{D})}$.

\section{Young Measures, Weak Limits, and Compensated Compactness}

Let $\Omega \subset \mathbb{R}^{N}$ be a bounded open set. Assume that a sequence of functions $\mathbf{u}^{\varepsilon}: \Omega \rightarrow \mathbb{R}^{m}$ satisfies

$$
\mathbf{u}^{\varepsilon} \stackrel{*}{\rightarrow} \mathbf{u} \in L^{\infty}(\Omega)
$$

and

$$
\sum_{j=1}^{N} A_{j} \frac{\partial \mathbf{u}^{\varepsilon}}{\partial x_{j}} \quad \text { is compact in } H_{\mathrm{loc}}^{-1}(\Omega)
$$

for some $n \times m$ matrices $A_{j}, j=1, \cdots, N$. Then (3.2) can be regarded as $n$ first-order differential equations for the $m$ unknowns. A mathematical question is whether, for some appropriate subsequence,

$$
f\left(\mathbf{u}^{\varepsilon}\right) \rightarrow f(\mathbf{u}) \quad \text { in the sense of distributions }
$$

for any smooth nonlinear function $f$. If (3.2) were elliptic, the weak convergence would be equivalent to the convergence in $L_{l o c}^{2}$. However, this is not the case in general. For example, $u^{\varepsilon}\left(y_{1}, y_{2}\right)=\sin \left(\frac{y_{1}+y_{2}}{\varepsilon}\right)$ and $f(u)=u^{2}$, and then it is well known that $u^{\varepsilon} \rightarrow 0$ and $\left(u^{\varepsilon}\right)^{2} \stackrel{*}{\rightarrow} \frac{1}{2} \neq 0^{2}$. Nevertheless, we have the following theorem.

Theorem 3.1 $([102,78])$. Let $\mathbf{u}^{\varepsilon}=\left(u_{1}^{\varepsilon}, u_{2}^{\varepsilon}, u_{3}^{\varepsilon}, u_{4}^{\varepsilon}\right): \Omega \subset \mathbb{R}^{2} \rightarrow \mathbb{R}^{4}$ be measurable functions satisfying

$$
\begin{aligned}
& w-\lim \mathbf{u}^{\varepsilon}=\mathbf{u} \quad \text { in } L^{2}(\Omega), \\
& \frac{\partial u_{1}^{\varepsilon}}{\partial y_{1}}+\frac{\partial u_{2}^{\varepsilon}}{\partial y_{2}}, \quad \frac{\partial u_{3}^{\varepsilon}}{\partial y_{1}}+\frac{\partial u_{4}^{\varepsilon}}{\partial y_{2}} \quad \text { are compact in } H_{\mathrm{loc}}^{-1}(\Omega) .
\end{aligned}
$$

Then there exists a subsequence (still labeled) $\mathbf{u}^{\varepsilon}$ such that

$$
\left|\begin{array}{ll}
u_{1}^{\varepsilon} & u_{2}^{\varepsilon} \\
u_{3}^{\varepsilon} & u_{4}^{\varepsilon}
\end{array}\right| \rightarrow\left|\begin{array}{ll}
u_{1} & u_{2} \\
u_{3} & u_{4}
\end{array}\right| \quad \text { in the sense of distributions. }
$$

The weak limits can be represented by a family of probability measures, Young measures, as described in the following theorem.

Theorem 3.2. Let $\Omega \subset \mathbb{R}^{N}$ be a Lebesgue measurable domain. Let $K \subset \mathbb{R}^{m}$ be closed (but not necessarily bounded). Let $\left\{\mathbf{u}^{\varepsilon}: \Omega \rightarrow \mathbb{R}^{m}\right\}_{\varepsilon>0}$ be a sequence of Lebesgue measurable functions satisfying

$$
\lim _{\varepsilon \rightarrow 0}\left|\left\{\mathbf{y} \in \Omega: \mathbf{u}^{\varepsilon}(\mathbf{y}) \notin V\right\}\right|=0
$$


for some neighborhood $V \subset \mathbb{R}^{m}$ of $K$. Then there exists a subsequence $\left\{\mathbf{u}^{\varepsilon_{j}}\right\}_{j=1}^{\infty} \subset$ $\left\{\mathbf{u}^{\varepsilon}\right\}_{\varepsilon>0}$ and a family of Young measures $\left\{\nu_{\mathbf{y}}\right\}_{\mathbf{y} \in \Omega}$ such that

(i) For a.e. $\mathbf{y} \in \Omega$,

$$
\nu_{\mathbf{y}} \geq 0, \quad \operatorname{supp} \nu_{\mathbf{y}} \subset K, \quad\left\|\nu_{\mathbf{y}}\right\|_{\mathcal{M}}:=\nu_{\mathbf{y}}\left(\mathbb{R}^{m}\right) \leq 1 \quad \text { a.e. } \mathbf{y} \in \Omega
$$

(ii) For any $f \in C\left(\mathbb{R}^{m} ; \mathbb{R}\right)$ satisfying $\lim _{|\lambda| \rightarrow \infty} f(\lambda)=0$,

$$
\begin{aligned}
& \left\langle\nu_{\mathbf{y}}(\lambda), f(\lambda)\right\rangle \quad \text { is measurable with respect to } \mathbf{y} \in \Omega, \\
& f\left(\mathbf{u}^{\varepsilon_{j}}\right) \stackrel{*}{\rightarrow}\left\langle\nu_{\mathbf{y}}, f\right\rangle=\int_{\mathbb{R}^{m}} f(\lambda) d \nu_{\mathbf{y}}(\lambda) \quad \text { in } L^{\infty} .
\end{aligned}
$$

Furthermore, if

$$
\lim _{k \rightarrow \infty} \sup _{j}\left|\left\{\mathbf{y} \in \Omega \cap B(0, R):\left|\mathbf{u}^{\varepsilon_{j}}(\mathbf{y})\right| \geq k\right\}\right|=0 \quad \text { for any } R>0,
$$

then

(i) $\left\|\nu_{\mathbf{y}}\right\|_{\mathcal{M}}=1 \quad$ a.e. $\mathbf{y} \in \Omega$;

(ii) For any measurable subset $A \subset \Omega$ and for any $f \in C\left(\mathbb{R}^{m} ; \mathbb{R}\right)$ such that

$$
f\left(\mathbf{u}^{\varepsilon_{j}}\right) \rightarrow\left\langle\nu_{\mathbf{y}}, f\right\rangle \quad \text { in } L^{1}(A)
$$

The notion of Young measures was first introduced by Young [109]. MacShane, [75], also introduced a related idea. When $\sup _{\varepsilon>0}\left\|\mathbf{u}^{\varepsilon}\right\|_{L^{\infty}}<\infty$, Theorem 3.2 was first established by Tartar [102], and an elementary proof can be found in [16]. The general form of Theorem 3.2 is due to Ball [6], where a detailed proof can be found. Also see Ambrosio-Fusco-Pallara [2] and Lin-Yang [69] for a more general treatment.

Remark 3.1. Condition (3.7) is very weak and equivalent to the following: Given $R>0$, there exists a nondecreasing function $g_{R} \in C([0, \infty) ; \mathbb{R})$ satisfying $\lim _{t \rightarrow \infty} g_{R}(t)=\infty$ such that

$$
\sup _{j} \int_{\Omega \cap B(0, R)} g_{R}\left(\left|\mathbf{u}^{\varepsilon_{j}}(\mathbf{y})\right|\right) d \mathbf{y}<\infty .
$$

Condition (3.8) is equivalent to the de la Valleé Poussin Criterion: There exists some $\psi \in C([0, \infty) ; \mathbb{R})$ with $\lim _{\lambda \rightarrow \infty} \frac{\psi(\lambda)}{\lambda}=\infty$ such that

$$
\sup _{0<\varepsilon \leq 1} \int_{\Omega} \psi\left(\left|\mathbf{u}^{\varepsilon}(\mathbf{y})\right|\right) d \mathbf{y}<\infty ;
$$


and is also equivalent to the Dunford-Pettis Criterion:

$$
\left\{\begin{array}{l}
\sup _{0<\varepsilon \leq 1}\left\|\mathbf{u}^{\varepsilon}\right\|_{L^{1}(\Omega)}<\infty, \\
\lim _{|E| \rightarrow 0, E \subset \Omega} \int_{E}\left|\mathbf{u}^{\varepsilon}(\mathbf{y})\right| d \mathbf{y}=0 \quad \text { uniformly for } 0<\varepsilon \leq 1 .
\end{array}\right.
$$

Remark 3.2. Let $\left\|\mathbf{u}^{\varepsilon}\right\|_{L^{p}(\Omega)} \leq C$ for some $p \in(1, \infty]$. Then Theorem 3.2 implies that there exists a family of Young measures $\left\{\nu_{\mathbf{y}}(\lambda) \in \operatorname{Prob}\left(\mathbb{R}^{m}\right)\right\}_{\mathbf{y} \in \Omega}$ and a subsequence $\left\{\mathbf{u}^{\varepsilon_{j}}\right\}_{j=1}^{\infty} \subset\left\{\mathbf{u}^{\varepsilon}\right\}_{\varepsilon>0}$ such that

(i) When $p=\infty$,

$$
f\left(\mathbf{u}^{\varepsilon_{j}}\right) \stackrel{*}{\rightarrow}\left\langle\nu_{\mathbf{y}}, f(\lambda)\right\rangle \quad \text { in } L^{\infty}(\Omega)
$$

for any function $f \in C\left(\mathbb{R}^{m} ; \mathbb{R}\right)$ (cf. Tartar [102]);

(ii) When $1<p<\infty$,

$$
\begin{aligned}
& f\left(\mathbf{u}^{\varepsilon_{j}}\right) \rightarrow\left\langle\nu_{\mathbf{y}}, f(\lambda)\right\rangle \quad \text { in } L^{r}(\Omega), r \in(1, p / q), \\
& \nu_{\mathbf{y}}(|\lambda|=\infty)=0 \quad \text { a.e. } \mathbf{y} \in \Omega,
\end{aligned}
$$

for any function $f \in C\left(\mathbb{R}^{m} ; \mathbb{R}\right)$ satisfying $|f(\lambda)| \leq C\left(1+|\lambda|^{q}\right)$ for $\lambda \in$ $R^{m}, q<p$ (see Schonbek [92]).

Remark 3.3. For any Lebesgue point $\mathbf{y} \in \mathbb{R}^{N}$, the Young measure $\nu_{\mathbf{y}}$ can be also understood as the limiting probability distribution of the values of $\left\{\mathbf{u}^{\varepsilon}(\mathbf{y})\right\}$ near the point $\mathbf{y}$ as $\varepsilon \rightarrow \infty$ (see Ball [6]). Set

$$
\left\langle\nu_{\mathbf{y}, r}^{\varepsilon}, f\right\rangle:=\frac{1}{|B(\mathbf{y}, r)|} \int_{B(\mathbf{y}, r)} f\left(\mathbf{u}^{\varepsilon}(\mathbf{x})\right) d \mathbf{x} \quad \text { for any } f \in C_{c}\left(\mathbb{R}^{m}\right) .
$$

Then $\left\|\nu_{\mathbf{y}, r}^{\varepsilon}\right\| \leq 1$. Thus there exist a subsequence $\left\{\varepsilon_{j}\right\}_{j=1}^{\infty}$ and a measure $\nu_{\mathbf{y}, r}$ such that $\nu_{\mathbf{y}, r}^{\varepsilon_{j}} \rightarrow \nu_{\mathbf{y}, r}$, i.e.,

$$
\left\langle\nu_{\mathbf{y}, r}, f\right\rangle=\frac{1}{|B(\mathbf{y}, r)|} \int_{B(\mathbf{y}, r)}\left\langle\nu_{\mathbf{x}}, f\right\rangle d \mathbf{x} .
$$

Since $\mathbf{y}$ is a Lebesgue point, we have

$$
\left\langle\nu_{\mathbf{y}, r}, f\right\rangle \rightarrow\left\langle\nu_{\mathbf{y}}, f\right\rangle \quad \text { when } r \rightarrow 0 .
$$

Remark 3.4. When $\operatorname{diam}\left(\operatorname{supp} \nu_{\mathbf{y}}\right)<\infty$, the deviation between the weak and strong convergence is measured by the spreading of the support of $\nu_{\mathbf{y}}$ : If $f$ is a Lipschitz continuous function, then

$$
\left\|f\left(\omega^{*}-\lim u^{\varepsilon}\right)-\omega^{*}-\lim f\left(\mathbf{u}^{\varepsilon}\right)\right\|_{L^{\infty}} \leq\|f\|_{\operatorname{Lip}} \sup _{\mathbf{y}}\left(\operatorname{diam}\left(\operatorname{supp} \nu_{\mathbf{y}}\right)\right) .
$$

As a corollary, we have 
Theorem 3.3. A uniformly bounded sequence $\mathbf{u}^{\varepsilon}$ converges to $\mathbf{u}$ a.e. in $\Omega$ if and only if the corresponding family of Young measures $\nu_{\mathbf{y}}$ reduces to a family of Dirac masses concentrated at $\mathbf{u}(\mathbf{y})$ at almost every point $\mathbf{y}$, i.e., $\nu_{\mathbf{y}}=\delta_{\mathbf{u}(\mathbf{y})}$ a.e.

The following compactness interpolation is useful for obtaining the $H_{l o c}^{-1}$-compactness needed as in Theorem 3.1.

Theorem 3.4. Suppose that $q>1$ and $r \in(q, \infty]$ are constants. Then

$$
\begin{aligned}
& \text { (compact set of } \left.W_{l o c}^{-1, q}(\Omega)\right) \cap\left(\text { bounded set of } W_{l o c}^{-1, r}(\Omega)\right) \\
& \subset\left(\text { compact set of } W_{l o c}^{-1, p}(\Omega)\right) \quad \text { for any } p \in[q, r) .
\end{aligned}
$$

This is a generalization of Murat's Lemma [102, 79], whose proof can be found in $[16,42]$.

Some more general results and detailed proofs can be found in Tartar [102, 103] and Murat [78, 79, 80]. Other related references include Chen [19], CoifmanLions-Meyer-Semmes [34], Dacorogna [36], Evans [46], Hörmander [63], Morrey [77], Struwe [100], Young [109], and the references cited therein.

The next issue is how the compactness required for constructing entropy solutions and their behavior can be achieved. As an example, from Theorem 3.4 and an idea in [17], we first have

Theorem 3.5 ([19]). Consider a hyperbolic system of conservation laws (1.1) with a strictly convex entropy pair $\left(\eta_{*}, \mathbf{q}_{*}\right)$. Assume that the uniformly bounded sequence $\mathbf{u}^{\varepsilon}(t, \mathbf{x}) \in L^{\infty}\left(\mathbb{R}_{+}^{d+1}\right)$ satisfies

$$
\partial_{t} \eta\left(\mathbf{u}^{\varepsilon}\right)+\nabla_{\mathbf{x}} \cdot \mathbf{q}\left(\mathbf{u}^{\varepsilon}\right) \leq 0 \quad \text { in the sense of distributions, }
$$

for any convex entropy pair $(\eta, \mathbf{q}) \in \Lambda$, where $\Lambda$ is a linear space of all (not necessarily convex) entropy pairs of (1.1) including $\left(\eta_{*}, \mathbf{q}_{*}\right)$. Then

$$
\partial_{t} \eta\left(\mathbf{u}^{\varepsilon}\right)+\nabla_{\mathbf{x}} \cdot \mathbf{q}\left(\mathbf{u}^{\varepsilon}\right) \quad \text { is compact in } W_{\text {loc }}^{-1, p}\left(\mathbb{R}_{+}^{d+1}\right), p \in(1, \infty),
$$

for any (not necessarily convex) entropy pair $(\eta, \mathbf{q}) \in \Lambda$ satisfying $\left|\nabla^{2} \eta\right| \leq$ $C \nabla^{2} \eta_{*}$

This theorem is designed to prove the compactness of solution operators in $L_{\text {loc }}^{1}\left(\mathbb{R}_{+}^{d+1}\right)$ by using the method of compensated compactness.

\section{Cauchy Fluxes, Balance Laws, and Divergence-Measure Fields}

In this section, we first discuss a natural connection between Cauchy fluxes and divergence-measure fields and then exhibit a general framework for the derivation of nonlinear systems of balance laws from the physical principle of balance law. 
4.1. Cauchy fluxes and divergence-measure fields. The physical principle of balance law with the form

$$
\int_{\partial \Omega} f(\mathbf{y}, \nu(\mathbf{y})) d \mathcal{H}^{N-1}(\mathbf{y})+\int_{\Omega} b(\mathbf{y}) d \mathbf{y}=0
$$

is basic in all of classical physics, where $\nu(\mathbf{y})$ is the interior unit normal on the boundary $\partial \Omega$ to $\Omega$. In mechanics, $f$ represents the surface force per unit area on $\partial \Omega$, while in thermodynamics $f$ gives the heat flow per unit area across the boundary $\partial \Omega$.

In 1823, Cauchy [14] established the Stress Theorem that is probably the most important theorem in continuum mechanics: If $f(\mathbf{y}, \nu(\mathbf{y}))$, defined for each $\mathbf{y}$ in an open region $\mathbb{D}$ and every unit vector $\nu$, is continuous in $\mathbf{y}$, if $b(\mathbf{y})$ is uniformly bounded on $\mathbb{D}$, and if (4.1) is satisfied for every smooth region $\Omega \subset \mathbb{D}$, then $f(\mathbf{y}, \nu)$ must be linear in $\nu$. The Cauchy Postulate states that the density flux $f$ through a surface depends on the surface solely through the normal at that point. For instance, if $f(\mathbf{y}, \nu)$ represents the heat flow, then the Stress Theorem says that there exists a vector field $\mathbf{F}$ such that

$$
f(\mathbf{y}, \nu)=\mathbf{F}(\mathbf{y}) \cdot \nu .
$$

Since the time of Cauchy's Stress Theorem [14], many efforts have been made to generalize his ideas and remove some of his hypotheses. The first results in this direction were obtained by Noll [83] in 1959, who set up a basis for an axiomatic foundation for continuum thermodynamics. In particular, Noll [83] showed that the Cauchy Postulate may directly follow from the balance law. In [59], Gurtin-Martins introduced the concept of Cauchy fluxes and removed the continuity assumption on $f$. In [111], Ziemer proved Noll's theorem in the context of geometric measure theory, in which the Cauchy fluxes were first formulated at the level of generality with sets of finite perimeter in the absence of jump surfaces, "shock waves". Motivated by these works, a more general framework has been formulated in Chen-Torres-Ziemer [32] for the Cauchy fluxes that allow the presence of these exceptional jump surfaces. Before introducing this framework, we need the following definitions.

Definition 4.1. An oriented surface is a pair $(S, \nu)$ so that $S \subset \mathbb{R}^{N}$ is a Borel set and $\nu: \mathbb{R}^{N} \rightarrow \mathbb{S}^{N-1}$ is a Borel measurable unit vector field that satisfy the following property: There is a bounded set $\Omega$ of finite perimeter such that $S \subset \partial^{*} \Omega$ (the measure-theoretic reduced boundary of $\Omega$ ) and

$$
\nu(\mathbf{y})=\nu_{\Omega}(\mathbf{y}) \chi_{S}(\mathbf{y}),
$$

where $\chi_{S}$ is the characteristic function of the set $S$ and $\nu_{\Omega}(\mathbf{y})$ is the interior measure-theoretic unit normal to $\Omega$ at $\mathbf{y}$.

Two oriented surfaces $\left(S_{j}, \nu_{j}\right), j=1,2$, are said to be compatible if there exists a set of finite perimeter $\Omega$ such that $S_{j} \subset \partial^{*} \Omega$ and $\nu_{j}(\mathbf{y})=\nu_{\Omega}(\mathbf{y}) \chi_{S_{j}}(\mathbf{y})$, 
$j=1,2$. For simplicity, we will denote the pair $(S, \nu)$ simply as $S$, with implicit understanding that $S$ is oriented by the interior normal of some set $\Omega$ of finite perimeter. We define $-S=(S,-\nu)$, which is regarded as a different oriented surface.

Definition 4.2. Let $\mathbb{D}$ be a bounded open set. A Cauchy flux is a functional $\mathcal{F}$ that assigns to each oriented surface $S:=(S, \nu) \Subset \mathbb{D}$ a real number and has the following properties:

(i) $\mathcal{F}\left(S_{1} \cup S_{2}\right)=\mathcal{F}\left(S_{1}\right)+\mathcal{F}\left(S_{2}\right)$ for any pair of compatible disjoint surfaces $S_{1}, S_{2} \Subset \mathbb{D}$

(ii) There exists a nonnegative Radon measure $\sigma$ such that

$$
\left|\mathcal{F}\left(\partial^{*} \Omega\right)\right| \leq \sigma(\Omega)
$$

for every set of finite perimeter $\Omega \Subset \mathbb{D}$ satisfying $\sigma(\partial \Omega)=0$;

(iii) There exists a constant $K$ such that

$$
|\mathcal{F}(S)| \leq K \mathcal{H}^{N-1}(S)
$$

for every oriented surface $S \Subset \mathbb{D}$ satisfying $\sigma(S)=0$.

This general framework for Cauchy fluxes allows the presence of exceptional surfaces, "shock waves", in the formulation of the axioms, on which the measure $\sigma$ has support. On these exceptional surfaces, the Cauchy flux $\mathcal{F}$ has a discontinuity and hence the relation $\mathcal{F}(S)=-\mathcal{F}(-S)$ does not hold. In fact, the exceptional surfaces are supported on the singular part of the measure $\sigma$. When $\sigma$ reduces to the $N$-dimensional Lebesgue measure $\mathcal{L}^{N}$, the formulation reduces to Ziemer's formulation and in this case $\sigma$ vanishes on any $\mathcal{H}^{N-1}$-dimensional surface, which excludes shock waves.

In this general framework, the nonnegative Radon measure $\sigma$ is included to allow to capture measure-valued production density in the formulation of the balance law and entropy dissipation for entropy solutions of hyperbolic conservation laws. Such a framework is fundamental for the derivation of nonlinear systems of balance laws with measure-valued source terms from the physical principle of balance law. The framework also allows the recovery of Cauchy entropy fluxes through the Lax entropy inequality for entropy solutions of hyperbolic conservation laws by capturing entropy dissipation.

It has been established in Chen-Torres-Ziemer [32] that the Cauchy flux defined above induces a divergence-measure (vector) field $\mathbf{F}$ with the property that the Cauchy flux over every oriented surface can be recovered through the normal trace of $\mathbf{F}$ to the oriented surface. More precisely, we have 
Theorem 4.1 (Chen-Torres-Ziemer [32]). Let $\mathcal{F}$ be a Cauchy flux. Then there exists a unique divergence-measure field $\mathbf{F} \in \mathcal{D} \mathcal{M}^{\infty}(\mathbb{D})$ such that

$$
\mathcal{F}(S)=-\int_{S}(\mathbf{F} \cdot \nu)_{i} d \mathcal{H}^{N-1}
$$

for every oriented surface $(S, \nu)$, where $(\mathbf{F} \cdot \nu)_{i}$ is the normal trace of $\mathbf{F}$ to the oriented surface.

We now explain how the Cauchy flux can be recovered on the exceptional surfaces. Let $(S, \nu)$ be an oriented surface on which $\mathcal{F}(-S) \neq-\mathcal{F}(S)$. By definition of oriented surfaces, there exists a bounded set $\Omega:=\Omega^{1} \cup \partial^{*} \Omega$ of finite perimeter such that

$$
S \subset \partial^{*} \Omega \quad \text { and } \quad \nu(\mathbf{y})=\nu_{\Omega}(\mathbf{y}) \chi_{S}(\mathbf{y}),
$$

where $\nu_{\Omega}(\mathbf{y})$ is the interior normal to $\Omega$ at $\mathbf{y} \in S$ and $\Omega^{1}$ is the measure-theoretic interior of $\Omega$. Consider

$$
\tilde{\Omega}=\Omega^{0} \cup \partial^{*} \Omega,
$$

where $\Omega^{0}$ is the measure-theoretic exterior of $\Omega$.

Then Theorem 2.4 implies that there exist the normal traces $(\mathbf{F} \cdot \nu)_{i}$ and $(\mathbf{F} \cdot \nu)_{e}$ defined on $\partial^{*} \tilde{\Omega}=\partial^{*} \Omega$ respectively such that

$$
\begin{aligned}
\int_{\Omega^{1}} \operatorname{div} \mathbf{F} & =-\int_{\partial^{*} \Omega}(\mathbf{F} \cdot \nu)_{i} d \mathcal{H}^{N-1} \\
\int_{\Omega} \operatorname{div} \mathbf{F} & =-\int_{\partial^{*} \tilde{\Omega}}(\mathbf{F} \cdot \nu)_{e} d \mathcal{H}^{N-1}=-\int_{\partial^{*} \Omega}(\mathbf{F} \cdot \nu)_{e} d \mathcal{H}^{N-1} .
\end{aligned}
$$

It has been proved in [32] that the traces $(\mathbf{F} \cdot \nu)_{i}$ and $(\mathbf{F} \cdot \nu)_{e}$ can be recovered, up to a set of arbitrary small $\mathcal{H}^{N-1}$-measure, from the neighborhood behavior of the vector field $\mathbf{F}$. This observation allows us to define

$$
\mathcal{F}(S)=\mathcal{F}(S, \nu):=-\int_{S}(\mathbf{F} \cdot \nu)_{i} d \mathcal{H}^{N-1}
$$

and

$$
\mathcal{F}(-S)=\mathcal{F}(S,-\nu):=-\int_{S}(\mathbf{F} \cdot(-\nu))_{e} d \mathcal{H}^{N-1}=\int_{S}(\mathbf{F} \cdot \nu)_{e} d \mathcal{H}^{N-1}
$$

In this way, we can recover the Cauchy flux $\mathcal{F}$ through the corresponding divergencemeasure field $\mathbf{F}$ over all oriented surfaces, especially including the exceptional surfaces. That is, the normal traces of $\mathbf{F} \in \mathcal{D} \mathcal{M}^{\infty}(\mathbb{D})$ are the Cauchy densities over all oriented surfaces. 
4.2. Systems of balance laws. Generally speaking, a balance law on an open subset $\mathbb{D}$ of $\mathbb{R}^{N}$ postulates that the production of a vector-valued "extensive" quantity in any bounded measurable subset $\Omega \subset \mathbb{D}$ of finite perimeter is balanced by the Cauchy flux of this quantity through the measure-theoretic reduced boundary $\partial^{*} \Omega$ of $\Omega$ (see Dafermos [36]).

Like the Cauchy flux, the production is introduced through a functional $\mathcal{P}$, defined on any bounded measurable subset $\Omega \subset \mathbb{D}$ of finite perimeter, taking value in $\mathbb{R}^{m}$ and satisfying the conditions:

$$
\begin{aligned}
& \mathcal{P}\left(\Omega_{1} \cup \Omega_{2}\right)=\mathcal{P}\left(\Omega_{1}\right)+\mathcal{P}\left(\Omega_{2}\right) \quad \text { if } \Omega_{1} \cap \Omega_{2}=\emptyset, \\
& |\mathcal{P}(\Omega)| \leq \sigma(\Omega) .
\end{aligned}
$$

Then the physical principle of balance law can be mathematically formulated as

$$
\mathcal{F}\left(\partial^{*} \Omega\right)=\mathcal{P}(\Omega)
$$

for any bounded measurable subset $\Omega \subset \mathbb{D}$ of finite perimeter. Conditions (4.6)(4.7) imply that there is a production density $\mathbf{P} \in \mathcal{M}\left(\mathbb{D} ; \mathbb{R}^{m}\right)$ such that

$$
\mathcal{P}(\Omega)=\int_{\Omega^{1}} \mathbf{P}(\mathbf{y})
$$

On the other hand, Theorem 4.1 yields that there exists $\mathbf{F} \in \mathcal{D} \mathcal{M}^{\infty}\left(\mathbb{D} ; \mathbb{R}^{N \times m}\right)$ such that

$$
\mathcal{F}\left(\partial^{*} \Omega\right)=-\int_{\partial^{*} \Omega}(\mathbf{F} \cdot \nu)(\mathbf{y}) d \mathcal{H}^{N-1}(\mathbf{y})=\int_{\Omega^{1}} \operatorname{div} \mathbf{F}(\mathbf{y})
$$

for any set $\Omega$ of finite perimeter.

Then (4.8)-(4.10) yields the following system of field equations

$$
\operatorname{div} \mathbf{F}(\mathbf{y})=\mathbf{P}(\mathbf{y})
$$

in the sense of measures on $\mathbb{D}$.

We assume that the state of the medium is described by a state vector field $\mathbf{u}$, taking value in an open subset $U$ of $\mathbb{R}^{m}$, which determines the flux density field $\mathbf{F}$ and the production density field $\mathbf{P}$ at the point $\mathbf{y} \in \mathbb{D}$ by the constitutive equations:

$$
\mathbf{F}(\mathbf{y}):=\mathbf{F}(\mathbf{u}(\mathbf{y}), \mathbf{y}), \quad \mathbf{P}(\mathbf{y}):=\mathbf{P}(\mathbf{u}(\mathbf{y}), \mathbf{y}),
$$

where $\mathbf{F}(\mathbf{u}, \mathbf{y})$ and $\mathbf{P}(\mathbf{u}, \mathbf{y})$ are given smooth functions defined on $U \times \mathbb{D}$.

Combining (4.11) with (4.12) leads to the quasilinear first-order system of partial differential equations

$$
\operatorname{div} \mathbf{F}(\mathbf{u}(\mathbf{y}), \mathbf{y})=\mathbf{P}(\mathbf{u}(\mathbf{y}), \mathbf{y}),
$$

which is called a system of balance laws (cf. [36]). 
If $\mathcal{P}=0$, the previous derivation yields

$$
\operatorname{div} \mathbf{F}(\mathbf{u}(\mathbf{y}), \mathbf{y})=0
$$

which is called a system of conservation laws. When the medium is homogeneous:

$$
\mathbf{F}(\mathbf{u}, \mathbf{y})=\mathbf{F}(\mathbf{u}),
$$

that is, $\mathbf{F}$ depends on $\mathbf{y}$ only through the state vector, then the system of conservation laws (4.14) becomes

$$
\operatorname{div} \mathbf{F}(\mathbf{u}(\mathbf{y}))=0 .
$$

In particular, when the coordinate system $\mathbf{y}$ is described by the time variable $t$ and the space variable $\mathbf{x}=\left(x_{1}, \cdots, x_{d}\right)$ :

$$
\mathbf{y}=\left(t, x_{1}, \cdots, x_{d}\right)=(t, \mathbf{x}), \quad N=d+1,
$$

and the flux density is written as

$$
\mathbf{F}(\mathbf{u})=\left(\mathbf{u}, \mathbf{f}_{1}(\mathbf{u}), \cdots, \mathbf{f}_{d}(\mathbf{u})\right)=(\mathbf{u}, \mathbf{f}(\mathbf{u})),
$$

then we have the standard form (1.1) for systems of conservation laws.

\section{Multidimensional Scalar Conservation LaWs}

In this section we consider (1.1) for the case $m=1$. That is,

$$
\partial_{t} u+\nabla_{\mathbf{x}} \cdot \mathbf{f}(u)=0, \quad \mathbf{x} \in \mathbb{R}^{d}, u \in \mathbb{R},
$$

with initial data

$$
\left.u\right|_{t=0}=u_{0}(\mathbf{x})
$$

5.1. Kinetic formulation and compactness of solution operators. Let $u=$ $u(t, \mathbf{x}) \in L^{\infty}$ be an entropy solution of (5.1) determined by (2.1). Set

$$
\chi(v ; u)=\left\{\begin{aligned}
+1 & \text { if } 0<v<u \\
-1 & \text { if } u<v<0 \\
0 & \text { otherwise }
\end{aligned}\right.
$$

Then it has been shown in Lions-Perthame-Tadmor [72] that there exists a locally finite Radon measure $\mu \in \mathcal{M}\left(\mathbb{R}_{v} \times \mathbb{R}_{t, \mathbf{x}}^{d+1}\right)$ such that $\chi(v ; u(t, \mathbf{x}))$ satisfies

$$
\partial_{t} \chi(v ; u(t, \mathbf{x}))+\mathbf{f}^{\prime}(v) \cdot \nabla_{\mathbf{x}} \chi(v ; u(t, \mathbf{x}))=\partial_{v} \mu(v ; t, \mathbf{x})
$$

in the sense of distributions in $\mathbb{R}_{+} \times \mathbb{R}^{d}$.

With the kinetic formulation (5.3), the following compactness theorem can be established by combining the Fourier analysis with averaging argument first in [72] (also see [23]). 
Theorem 5.1. Assume that $\mathbf{f} \in C^{1}\left(\mathbb{R}^{\prime} \mathbb{R}^{d}\right)$ satisfies that, for every $(\tau, \xi) \in(\mathbb{R} \times$ $\left.\mathbb{R}^{d}\right) \backslash\{(0,0)\}$,

$$
\mathcal{H}^{1}\left(\left\{v: \tau+\xi \cdot \mathbf{f}^{\prime}(v)=0\right\}\right)=0 .
$$

Then the solution operator $S_{t}: L^{\infty} \rightarrow L_{l o c}^{1}\left(\mathbb{R}_{+}^{d+1}\right)$, determined by $u(t, \cdot)=S_{t} u_{0}(\cdot)$ to (5.1)-(5.2), is compact.

Remark 5.1. A flux function $\mathbf{f}(v)$ that satisfies condition (5.4) is called genuinely nonlinear. The genuinely nonlinear condition (5.4) excludes the flux functions whose restriction to an open interval is linear; that is, $\mathbf{f}^{\prime}(v)$ is not contained, for any $v$-open interval, in a $(d-1)$-dimensional subspace. For the one-dimensional case, condition (5.4) is stronger than the following condition: There is no open interval on which the flux function $f(u)$ is affine, which also implies compactness (see Tartar [102] and Chen-Lu [28]).

5.2. Decay of periodic solutions. Combining the compactness theorem (Theorem 5.1) and entropy analysis with measure-theoretic analysis and scaling arguments, we have

Theorem 5.2 (Chen-Frid [23]). Let $u(t, \mathbf{x}) \in L^{\infty}\left(\mathbb{R}_{+}^{d+1}\right)$ be a periodic entropy solution of (5.1)-(5.2) in $\mathbb{R}_{+}^{d+1}$ with initial data $u_{0}$ whose period is $P$. Assume that condition (5.4) holds. Then

$$
\underset{t \rightarrow \infty}{\operatorname{ess}} \lim \|u(t, \cdot)-\bar{u}\|_{L^{1}(P)} \rightarrow 0
$$

with $\bar{u}=\frac{1}{|P|} \int_{P} u_{0}(\mathbf{x}) d \mathbf{x}$.

5.3. Initial-boundary value problem and boundary traces of entropy solutions. The connection between the strong trace of entropy solutions and the nonlinearity of the flux functions was first observed in Chen-Rascle [30] for the one-dimensional case. The connection between the blow-up elliptic techniques and hyperbolic theory was first noticed by Vasseur [104]. In [104], Vasseur constructed the blow-ups within the context of kinetic formulation (5.3) and used the results of existence and uniqueness of normal traces of divergence-measure fields in Chen-Frid [22] to establish the existence of one-sided strong traces (in the $L^{1}$ sense) for entropy solutions only in $L^{\infty}$. The kinetic formulation allows the use of the so-called averaging lemmas $[45,56]$ which hold under condition (5.4). More precisely, the result reads

Theorem 5.3 (Vasseur [104]). Let $\Omega \subset \mathbb{R}_{+}^{d+1}$ be an open set with deformable Lipschitz boundary. Assume that $\mathbf{f} \in C^{3}\left(\mathbb{R} ; \mathbb{R}^{d}\right)$ satisfies (5.4). Then, for every entropy solution $u \in L^{\infty}(\Omega ; \mathbb{R})$ of $(5.1)$, there exists $u^{\tau} \in L^{\infty}\left(\partial \Omega, \mathcal{H}^{d}\right)$ such that, for every regular Lipschitz deformation $\Psi$ of $\partial \Omega$ and every compact set $K \Subset \partial \Omega$,

$$
\underset{s \rightarrow 0}{\operatorname{ess} \lim } \int_{K}\left|u(\Psi(s,(t, \mathbf{x})))-u^{\tau}(t, \mathbf{x})\right| d \mathcal{H}^{d}(t, \mathbf{x})=0 .
$$


In particular, for every continuous function $g(u)$,

$$
[g(u)]^{\tau}=g\left(u^{\tau}\right)
$$

A corresponding result may also hold without the nonlinearity condition (5.4), which was recently observed in Panov [84] based on the $H$-measure idea and Kwon-Vasseur [65] based on the blow-up idea.

5.4. Structure of entropy solutions in $L^{\infty}$. Another application of the blow-up techniques within the context of kinetic formulation (5.3) is the recent work by De Lellis-Otto-Westdickenberg [40] for entropy solutions to (5.1) only in $L^{\infty}$. In [40], the classification of geometric blow-ups (the technique is similar to that used to obtain the regularity of minimal surfaces) and geometric-measure arguments are employed in order to obtain the regularity properties of entropy solutions of multidimensional scalar conservation laws. The regularizing mechanism comes from the fact that the flux function $\mathbf{f}(v)$ satisfies (5.4), i.e., $\mathbf{f}(v)$ is genuinely nonlinear. The solutions are shown to have a $B V$-like structure although they do not have bounded variation. This is achieved in the context of kinetic formulation (5.3), which allows the use of compactness results for velocity averages [45, 56, 85].

Theorem 5.4 (De Lellis-Otto-Westdickenberg [40]). If $\mathbf{f}$ satisfies (5.4) and $u$ is an entropy solution of (5.1), then there exists a rectifiable set $J$ of dimension $d$ such that

(i) $u$ has vanishing mean oscillation at every $(t, \mathbf{x}) \notin J$, that is,

$$
\lim _{r \rightarrow 0} \frac{1}{r^{d+1}} \int_{B_{r}(t, \mathbf{x})}\left|u(\tau, \mathbf{y})-\bar{u}_{(t, \mathbf{x}), r}\right| d \mathbf{y} d \tau=0,
$$

where $\bar{u}_{(t, \mathbf{x}), r}$ denotes the average of $u$ on the ball $B_{r}(t, \mathbf{x})$;

(ii) $u$ has strong left and right traces on $J$, that is, there exist functions $u^{-}, u^{+}$: $J \rightarrow \mathbb{R}$ such that, for $\mathcal{H}^{d}-$ a.e. $(t, \mathbf{x}) \in J$,

$\lim _{r \rightarrow 0} \frac{1}{r^{d+1}}\left\{\int_{B_{r}^{-}(t, \mathbf{x})}\left|u(\tau, \mathbf{y})-u^{-}(t, \mathbf{x})\right| d \mathbf{y} d \tau+\int_{B_{r}^{+}(t, \mathbf{x})}\left|u(\tau, \mathbf{y})-u^{+}(t, \mathbf{x})\right| d \mathbf{y} d \tau\right\}=0$, where $B_{r}^{ \pm}:=\left\{(\tau, \mathbf{y}) \in B_{r}(t, \mathbf{x}): \pm(\tau-t, \mathbf{y}-\mathbf{x}) \cdot \nu(t, \mathbf{x})>0\right\}$ for the unit normal $\nu$ to $J$.

The analysis of geometric blow-ups (around the points of $J$ ) of $u$ and $\mu$ in the kinetic formulation (5.3) yields Theorem 5.4. These blow-ups are the limits of subsequences of $u^{r}$ and $\mu^{r}$ as $r \rightarrow 0$, where $u^{r}(\tau, \mathbf{y})=u(t+r \tau, \mathbf{x}+r \mathbf{y})$ and $\mu^{r}(B \times A)=\frac{1}{r^{d}} \mu(B \times((t, \mathbf{x})+r A))$ for $A \subset \mathbb{R}^{d+1}$ and $B \subset \mathbb{R}$. 


\section{Systems of Conservation Laws}

In this section we consider (1.1) for the case $d=1$. That is,

$$
\partial_{t} \mathbf{u}+\partial_{x} \mathbf{f}(\mathbf{u})=0, \quad x \in \mathbb{R}, \mathbf{u} \in \mathbb{R}^{m},
$$

with initial data

$$
\left.\mathbf{u}\right|_{t=0}=\mathbf{u}_{0}(x)
$$

6.1. Existence, compactness, asymptotic behavior of entropy solutions. Assume that a sequence of functions $\mathbf{u}^{\varepsilon}$ satisfies the following conditions:

(i) $L^{\infty}$-bound:

$$
\left\|\mathbf{u}^{\varepsilon}\right\|_{L^{\infty}} \leq C
$$

(ii) For $C^{2}$ entropy pairs $(\eta, q) \in \Lambda_{1}$ (not necessarily convex),

$$
\partial_{t} \eta\left(\mathbf{u}^{\varepsilon}\right)+\partial_{x} q\left(\mathbf{u}^{\varepsilon}\right) \quad \text { is compact in } H_{\text {loc }}^{-1}\left(\mathbb{R}_{+}^{2}\right),
$$

where $\Lambda_{1}$ is a linear space of $C^{2}$ entropy pairs $(\eta, q)$ as solutions of the linear hyperbolic system $\nabla q=\nabla \eta \nabla \mathbf{f}$.

Then the question is whether $\mathbf{u}^{\varepsilon}(t, x) \rightarrow \mathbf{u}(t, x)$ a.e. when $\varepsilon \rightarrow 0$.

From Theorem 3.2, there exist a family of Young measures $\left\{\nu_{t, x}(\lambda)\right\}_{(t, x) \in \mathbb{R}_{+}^{2}}$ and a subsequence $\left\{\varepsilon_{k}\right\}_{k=1}^{\infty}$ such that

$$
\begin{aligned}
& \left(\eta_{j}\left(\mathbf{u}^{\varepsilon_{k}}(t, x)\right), q_{j}\left(\mathbf{u}^{\varepsilon_{k}}(t, x)\right)\right) \stackrel{*}{\rightarrow}\left(\left\langle\nu_{t, x}, \eta_{j}(\lambda)\right\rangle,\left\langle\nu_{t, x}, q_{j}(\lambda)\right\rangle\right), \quad j=1,2, \\
& \left|\begin{array}{l}
\eta_{1}\left(\mathbf{u}^{\varepsilon_{k}}(t, x)\right) q_{1}\left(\mathbf{u}^{\varepsilon_{k}}(t, x)\right) \\
\eta_{2}\left(\mathbf{u}^{\varepsilon_{k}}(t, x)\right) q_{2}\left(\mathbf{u}^{\varepsilon_{k}}(t, x)\right)
\end{array}\right| \rightarrow\left\langle\nu_{t, x},\left|\begin{array}{l}
\eta_{1}(\lambda) q_{1}(\lambda) \\
\eta_{2}(\lambda) q_{2}(\lambda)
\end{array}\right|\right\rangle \quad \text { when } k \rightarrow \infty .
\end{aligned}
$$

Then we obtain from Theorem 3.1 that the Young measures $\nu_{t, x}$ are governed by the static relation:

$$
\left\langle\nu_{t, x},\left|\begin{array}{l}
\eta_{1}(\lambda) q_{1}(\lambda) \\
\eta_{2}(\lambda) q_{2}(\lambda)
\end{array}\right|\right\rangle=\left|\begin{array}{l}
\left\langle\nu_{t, x}, \eta_{1}(\lambda)\right\rangle\left\langle\nu_{t, x}, q_{1}(\lambda)\right\rangle \\
\left\langle\nu_{t, x}, \eta_{2}(\lambda)\right\rangle\left\langle\nu_{t, x}, q_{2}(\lambda)\right\rangle
\end{array}\right|
$$

for any $C^{2}$ entropy pairs $\left(\eta_{j}, q_{j}\right) \in \Lambda_{1}, j=1,2$.

Furthermore, assume that the sequence $\mathbf{u}^{\varepsilon}(t, x)$ additionally satisfies that, for some $C^{2}$ entropy pair $(\eta, q)$ in a subclass $\Lambda_{2}$ of entropy pairs,

$$
\partial_{t} \eta\left(\mathbf{u}^{\varepsilon}\right)+\partial_{x} q\left(\mathbf{u}^{\varepsilon}\right) \leq o_{\varepsilon}(1)
$$

in the sense of distributions. Then the Young measures are also governed by the following dynamic entropy inequality:

$$
\partial_{t}\left\langle\nu_{t, x}, \eta(\lambda)\right\rangle+\partial_{x}\left\langle\nu_{t, x}, q(\lambda)\right\rangle \leq 0
$$

in the sense of distributions when the entropy pair $(\eta, q)$ lies in the class $\Lambda_{2}$. 
To prove $\mathbf{u}^{\varepsilon}(t, x) \rightarrow \mathbf{u}(t, x)$ a.e., it suffices, according to Theorem 3.3, to show that $\nu_{t, x}$ reduces to the Dirac mass $\delta_{\mathbf{u}(t, x)}(\lambda)$ for a.e. $(t, x)$ using only (6.5), or both (6.5) and (6.6).

For linear hyperbolic conservation laws, relation (6.5) is trivial, but the dynamic inequality (6.6) provides the main information on the Young measures $\nu_{t, x}$ depending on whether the initial data is oscillatory or not, while the weak convergence is enough to pass the limit in the linear equations. However, for nonlinear hyperbolic conservation laws, relation (6.5) indeed represents an imbalance of regularity; the operator on the left is more regular than one on the right due to cancellation, which provides additional information on $\nu_{t, x}$. The additional information on $\nu_{t, x}$ in (6.5) can force $\nu_{t, x}$ to be a point mass, for some examples of hyperbolic conservation laws with strong nonlinearity as assumed in the subsections below. The compactness framework is particularly useful for establishing the existence and compactness of entropy solutions without bounded variation.

Another important problem in the theory of hyperbolic systems of conservation laws is the decay of periodic entropy solutions, which is an important nonlinear phenomenon. For the linear case, initial oscillations propagate without decay. However, this is not the case for systems with certain nonlinearity as for the scalar case (Theorem 5.2). The Glimm-Lax theory [55] indicates the decay of periodic solutions obtained through Glimm's method [54] for $2 \times 2$ strictly hyperbolic and genuinely nonlinear systems for initial data of small oscillation. Using the generalized characteristics, Dafermos [38] showed for such systems that any periodic entropy solution, with local bounded variation and small oscillation, asymptotically decays.

With the help of the compactness framework, a new analytic approach has been developed in Chen-Frid [23] to study the decay of $L^{\infty}$ periodic solutions of hyperbolic systems of conservation laws via the compactness of solution operators. This was achieved without restrictions of either smallness or local bounded variation on the $L^{\infty}$ periodic initial data and any specific reference to a particular method for constructing the entropy solutions. Then we have the following decay framework.

Theorem 6.1 (Chen-Frid [23]). Consider system (6.1) endowed with a strictly convex entropy $\eta_{*}(\mathbf{u})$. Assume that $\mathbf{u}(t, x) \in L^{\infty}\left(\mathbb{R}_{+}^{2}\right)$ is an entropy solution of (6.1)-(6.2), which is periodic in $x$ with period $P$. Let the solution operator $S_{t}: L^{\infty} \rightarrow L_{l o c}^{1}\left(\mathbb{R}_{+}^{2}\right)$ determined by $\mathbf{u}(t, \cdot)=S_{t} \mathbf{u}_{0}(\cdot)$ is compact. Then the periodic solution $\mathbf{u}(t, x)$ asymptotically decays in $L^{1}$ to the average $\overline{\mathbf{u}}$ of its initial data over the period:

$$
\underset{t \rightarrow \infty}{\operatorname{ess} \lim _{P}} \int_{P}|\mathbf{u}(t, x)-\overline{\mathbf{u}}| d x \rightarrow 0
$$


with $\overline{\mathbf{u}}=\frac{1}{|P|} \int_{P} \mathbf{u}_{0}(x) d x$.

In Chen-Frid [23], an analytic framework for the asymptotic stability of nonperiodic entropy solutions has been also introduced with the aid of the theory of divergence-measure fields and the compactness framework established as below in this section.

Now we give several important examples to show how the compactness framework can be established, that is, the compactness of the sequence of functions $\mathbf{u}^{\varepsilon}(t, x)$ satisfying conditions (6.3)-(6.4).

6.2. Strictly hyperbolic systems with $m=2$. System (6.1) with $m=2$ is called strictly hyperbolic if $\lambda_{1}(\mathbf{u})<\lambda_{2}(\mathbf{u})$ with the right eigenvector $\mathbf{r}_{j}(\mathbf{u})$ corresponding to the $j$ th eigenvalue $\lambda_{j}(\mathbf{u})$ of $\nabla \mathbf{f}(\mathbf{u}), j=1,2$. We call $w_{i}(\mathbf{u}), i=$ 1,2 , Riemann invariants if they satisfy $\nabla w_{i}(\mathbf{u}) \cdot \mathbf{r}_{j}(\mathbf{u})=0, i \neq j$. Then we have $\nabla q \cdot \mathbf{r}_{j}=\lambda_{j} \nabla \eta \cdot \mathbf{r}_{j}$, which implies

$$
q_{w_{j}}=\lambda_{j}\left(w_{1}, w_{2}\right) \eta_{w_{j}}, \quad j=1,2 .
$$

Therefore, the entropy $\eta$ as a function of $\left(w_{1}, w_{2}\right)$ satisfies the following equation:

$$
\eta_{w_{1} w_{2}}+\frac{\lambda_{2 w_{1}}\left(w_{1}, w_{2}\right)}{\lambda_{2}-\lambda_{1}} \eta_{w_{2}}-\frac{\lambda_{1 w_{2}}\left(w_{1}, w_{2}\right)}{\lambda_{2}-\lambda_{1}} \eta_{w_{1}}=0
$$

Theorem 6.2 (DiPerna [44]). Assume that system (6.1) is strictly hyperbolic and purely genuinely nonlinear:

$$
\nabla \lambda_{j}(\mathbf{u}) \cdot \mathbf{r}_{j}(\mathbf{u}) \neq 0, \quad j=1,2 .
$$

If a sequence of functions $\mathbf{u}^{\varepsilon}(t, x)$ satisfies

(i) $\mathbf{u}^{\varepsilon}(t, x) \in K$ a.e., where $K \Subset \mathbb{R}^{2}$ is a compact subset of $\mathbb{R}^{2}$;

(ii) For any $C^{2}$ entropy pair $(\eta, q)$,

$$
\partial_{t} \eta\left(\mathbf{u}^{\varepsilon}\right)+\partial_{x} q\left(\mathbf{u}^{\varepsilon}\right) \quad \text { is compact in } H_{\text {loc }}^{-1}\left(\mathbb{R}_{+}^{2}\right) .
$$

Then $\mathbf{u}^{\varepsilon}(t, x)$ is compact in $L_{\text {loc }}^{1}\left(\mathbb{R}_{+}^{2}\right)$. In particular, the entropy solution operator is compact in $L_{l o c}^{1}\left(\mathbb{R}_{+}^{d+1}\right)$.

Now we consider the p-system in elastodynamics:

$$
\left\{\begin{array}{l}
\partial_{t} \tau-\partial_{x} v=0, \\
\partial_{t} v+\partial_{x} p(\tau)=0,
\end{array}\right.
$$

where $p(\tau) \in C^{2}$ with $p^{\prime}(\tau)<0$ satisfies that

(i) $\operatorname{sgn}\left((\tau-\hat{\tau}) p^{\prime \prime}(\tau)\right) \leq 0$ for some constant $\hat{\tau}$;

(ii) There is no interval on which $p$ is affine; 
(iii) There exists an integer $k \in[1, \infty]$ such that, on an interval $(\hat{\tau}, \hat{\tau}+\delta)$ or $(\hat{\tau}-\delta, \hat{\tau})$ for some $\delta>0$,

$$
\sum_{l=1}^{k}\left|p^{(2 l)}(\tau)\right| \neq 0 .
$$

Notice that, for this system, there are bounded invariant regions for entropy solutions. Then we have

Theorem 6.3 (Chen-Li-Li [27]). The result of Theorem 6.2 still holds for the p-system (6.10) satisfying (i)-(iii), although the purely genuine nonlinearity (6.9) fails when $\tau$ is in a set of measure zero. Furthermore, given any initial data in $L^{\infty}$, there exists a global entropy solution, and the corresponding solution operator is compact in $L_{l o c}^{1}\left(\mathbb{R}_{+}^{2}\right)$.

The special cases for Theorem 6.3 were first established by DiPerna [44] and Gripenberg [58]. Theorem 6.3 was proved in Chen-Li-Li [27] by identifying new properties of the Lax entropies, especially the higher-order terms in the Lax entropy expansions, and by developing new ways to employ these properties in the method of compensated compactness.

For the structure of Young measures for $2 \times 2$ strictly hyperbolic systems with linear degeneracy, see Serre [93] and Perthame-Tzavaras [87] for the Young measure reduction from (6.5). Also see Chen $[17,18]$ for the method of quasidecouping by using the dynamic inequality (6.6) and the initial information of the Young measures $\nu_{t, x}$, in addition to the reduction from (6.5).

6.3. Isentropic Euler equations. The system of isentropic Euler equations for a compressible fluid reads

$$
\left\{\begin{array}{l}
\partial_{t} \rho+\partial_{x} m=0 \\
\partial_{t} m+\partial_{x}\left(\frac{m^{2}}{\rho}+p(\rho)\right)=0
\end{array}\right.
$$

where the pressure $p(\rho)$ is a given nonlinear function of the density $\rho \geq 0$, determined by the fluid under consideration. The density $\rho \geq 0$ and mass $m$ are physically restricted by $|m| \leq C \rho$ for some constant $C>0$ so that the function $(\rho, m) \rightarrow m^{2} / \rho$ remains Lipschitz continuous, even at the vacuum $\rho=0$. For $\rho>0$, the velocity $v=m / \rho$ is uniquely defined. Strict hyperbolicity and purely genuine nonlinearity away from the vacuum for (6.11) require that the pressure law satisfy

$$
p^{\prime}(\rho)>0, \quad 2 p^{\prime}(\rho)+\rho p^{\prime \prime}(\rho)>0 \quad \text { for } \quad \rho>0 .
$$

At the vacuum, the two characteristic speeds of (3.1) may coincide and the systems be nonstrictly hyperbolic. A simple calculation shows that in general the vacuum can not be avoided for this system even for some Riemann solutions with 
large Riemann initial data away from the vacuum. This system is a prototype of nonlinear hyperbolic conservation laws in fluid mechanics.

The polytropic perfect gas is described by the equation of state:

$$
p_{*}(\rho)=\kappa \rho^{\gamma}, \quad \gamma>1 .
$$

One may choose $\kappa=(\gamma-1)^{2} /(4 \gamma)$, which is a convenient normalization. For early results on the existence of global entropy solutions of (6.11), we refer to Riemann [89] for the Riemann problem, Zhang-Guo [110] and Ding-Chang-Wang-Hsiao-Li [41] for a special class of initial data with bounded variation, Glimm [54] for arbitrary initial data with small total variation (also see Liu [73]), and NishidaSmoller [82] for large total variation but small $\gamma-1$ or vice versa by using the Glimm scheme [54]. For the limit case $\gamma=1$, Nishida [81] first established the existence of global solutions in $B V$ for large $B V$ initial data. For arbitrarily large $L^{\infty}$ initial data, the case $\gamma=1+2 / N(N \geq 5$ odd) was first treated by DiPerna [43]. The case $\gamma \in(1,5 / 3]$, which is the natural interval of $\gamma$ for the polytropic gas, was first completed in Ding-Chen-Luo [42] and Chen [15].

As discussed in $\S 3$, to achieve the compactness framework, it suffices to prove that the corresponding Young measures $\nu_{t, x}$, governed by $(6.5)$ for a.e. $(t, x)$ and for any two weak entropy pairs $\left(\eta_{j}, q_{j}\right), j=1,2$, are Dirac masses in the $(\rho, m)$ plane for our case. This is implied by the fact that the support of any Young measure in the $(\rho, v)$-plane, still denoted by $\nu_{t, x}$, is either a single point or a subset of the vacuum line $\{(\rho, v): \rho=0, v \in \mathbb{R}\}$. One of the main difficulties to reduce the Young measure here is that the commutation relation (6.5) holds only for weak entropy pairs (i.e., $\left.\eta\right|_{\rho=0}=0$ ), which is not allowed for any $C^{2}$ entropy pairs (different from those in Theorems 6.2-6.3), because of the degeneracy of the system near the vacuum.

When (6.13) holds, the weak entropies of (6.11) can be expressed by convolution of an arbitrary smooth function $\psi(s)$ and the entropy kernel, $\chi_{*}(\rho, v, s)$, defined by

$$
\chi_{*}(\rho, v ; s)=M_{*}\left[\rho^{\gamma-1}-(v-s)^{2}\right]_{+}^{\lambda}, \quad \lambda=\frac{3-\gamma}{2(\gamma-1)} .
$$

Here $y_{+}=\max (y, 0)$ and $M_{*}>0$ is the constant of normalization. Namely, one has $\eta(\rho, \rho v):=\int_{\mathbb{R}} \chi_{*}(\rho, v ; s) \psi(s) d s$. The entropy flux kernel has also an explicit form: $\sigma_{*}:=(v+\theta(s-v)) \chi_{*}$.

In the proof of DiPerna [43], Ding-Chen-Luo [42], and Chen [15], the heart of the matter is to construct special functions $\psi$ in order to exploit the set of constraints (6.5). These test functions are suitable approximations of high-order derivatives of the Dirac mass. In DiPerna [43], the case that $\lambda \geq 2$ is an integer was treated, in which all weak entropies are polynomial functions of the Riemann invariants. The idea of applying the technique of fractional derivatives was first 
introduced in Chen [15] and Ding-Chen-Luo [42] in order to deal with real values of $\lambda$.

Lions-Perthame-Souganidis [70] and Lions-Perthame-Tadmor [71], motivated by a kinetic formulation for (6.11) and (6.13), made the observation that the use of $\psi$ could be bypassed and (6.5) may be directly expressed by the entropy kernel $\chi_{*}(s)=\chi_{*}(\rho, v ; s)$ and the entropy flux kernel $\sigma_{*}(s)=\sigma_{*}(\rho, v ; s)$. That is, for all $s_{1}$ and $s_{2}$,

$$
\begin{aligned}
& \left\langle\nu_{t, x}, \chi_{*}\left(\rho, v ; s_{1}\right) \sigma_{*}\left(\rho, v ; s_{2}\right)-\chi_{*}\left(\rho, v ; s_{2}\right) \sigma_{*}\left(\rho, v ; s_{1}\right)\right\rangle \\
& =\left\langle\nu_{t, x}, \chi_{*}\left(\rho, v ; s_{1}\right)\right\rangle\left\langle\nu_{t, x}, \sigma_{*}\left(\rho, v ; s_{2}\right)\right\rangle-\left\langle\nu_{t, x}, \chi_{*}\left(\rho, v ; s_{2}\right)\right\rangle\left\langle\nu_{t, x}, \sigma_{*}\left(\rho, v ; s_{1}\right)\right\rangle .
\end{aligned}
$$

A simpler proof of the reduction of the Young measures satisfying (6.15) was given in [70] for the case $\gamma \in(1,3)$. The range $\gamma \in[3, \infty)$ was treated in [71]. The previous techniques of choosing suitable approximations of the derivatives of the Dirac mass, in $[15,42,43]$, correspond in their approach to computing a suitable number of $s$-derivatives of the commutation equation (6.15), the number of fractional derivatives being related to the exponent $\lambda$ which characterizes the singularities of the entropy kernel. This is technically delicate since such derivatives of the kernel generate Dirac masses, due to its limited regularity. It was observed that the average $\left\langle\nu, \chi_{*}\right\rangle$ is smoother (as a function of $s$ ) than $\chi_{*}(\rho, v, s$ ) (as a function of $(\rho, v, s))$.

A compactness framework for approximate solutions of this system with a general pressure law for large initial data has been established in [25]. The pressure function $p(\rho)$ satisfies that there exists a sequence of exponents

$$
1<\gamma:=\gamma_{1}<\gamma_{2}<\ldots<\gamma_{N} \leq(3 \gamma-1) / 2<\gamma_{N+1}
$$

and a sufficiently smooth function $P=P(\rho)$ such that

$$
\begin{aligned}
& p(\rho)=\sum_{n=1}^{N} \kappa_{n} \rho^{\gamma_{n}}+\rho^{\gamma_{N+1}} P(\rho), \\
& P(\rho) \text { and } \rho^{3} P^{\prime \prime \prime}(\rho) \text { are bounded as } \rho \rightarrow 0,
\end{aligned}
$$

for some coefficients $\kappa_{n} \in \mathbb{R}$ with $\kappa_{1}>0$. The solutions under consideration will remain in a bounded subset of $\{\rho \geq 0\}$ so that the behavior of $p(\rho)$ for large $\rho$ is irrelevant. This means that the pressure law $p(\rho)$ has the same singularity as $\sum_{n=1}^{N} \kappa_{n} \rho^{\gamma_{n}}$ near the vacuum. Observe that $p(0)=p^{\prime}(0)=0$, but, for $k>\gamma_{1}$, the higher derivative $p^{(k)}(\rho)$ is unbounded near the vacuum with different orders of singularity.

Consider system (6.11) with the general pressure law (6.12) and (6.16)-(6.18) with Cauchy data:

$$
\left.(\rho, m)\right|_{t=0}=\left(\rho_{0}, m_{0}\right)(x)
$$


Theorem 6.4 (Chen-LeFloch [25]). Assume that a sequence of functions $\left(\rho^{\varepsilon}, m^{\varepsilon}\right)$ satisfies that

(i) There exists some $C>0$ independent of $\varepsilon$ such that

$$
0 \leq \rho^{\varepsilon}(t, x) \leq C, \quad\left|m^{\varepsilon}(t, x)\right| \leq C \rho^{\varepsilon}(t, x) \quad \text { for a.e. }(t, x) ;
$$

(ii) For any weak entropy pair $(\eta, q)$ of (6.11)-(6.12) and (6.16)-(6.18),

$$
\partial_{t} \eta\left(\rho^{\varepsilon}, m^{\varepsilon}\right)+\partial_{x} q\left(\rho^{\varepsilon}, m^{\varepsilon}\right) \quad \text { is compact in } H_{\text {loc }}^{-1}\left(\mathbb{R}_{+}^{2}\right) \text {. }
$$

Then the sequence $\left(\rho^{\varepsilon}, m^{\varepsilon}\right)$ is compact in $L_{l o c}^{1}\left(\mathbb{R}_{+}^{2}\right)$.

Since (6.13) is not assumed, the explicit formula (6.14) is no longer available. In particular, the entropy flux kernel, denoted by $\sigma$, cannot be directly expressed from the entropy kernel, denoted by $\chi$. It turns out that several crucial steps of the proofs in the previous references can no longer be carried out.

The first step is to construct the entropy kernel $\chi$ and the entropy flux kernel $\sigma$. From (6.8), the entropy kernel is governed by a highly singular equation of Euler-Poisson-Darboux type:

$$
\chi_{w z}-\frac{\Lambda(w-z)}{w-z}\left(\chi_{w}-\chi_{z}\right)=0
$$

or, equivalently,

$$
\partial_{\rho \rho} \chi-k^{\prime}(\rho)^{2} \partial_{v v} \chi=0
$$

with initial data: $\left.\chi\right|_{\rho=0}=0,\left.\chi_{\rho}\right|_{\rho=0}=\delta_{v=s}$, involving a Dirac mass. Notice that the function $\Lambda(w-z)$ is not smooth in $w-z$ in general and that each of its derivatives produces certain extra powers of $1 /(w-z)$, which is singular near $w-z \approx 0$, that is, $\rho \approx 0$. Therefore, equation (6.22) or (6.23) is much more singular than the classical Euler-Poisson-Darboux equation, which one obtains for the $\gamma$-law (6.13). New techniques were developed and special care was taken to study the singularities of their derivatives. One of the major observations is that the principal singularities of $\chi$ and $\sigma$ can be determined (rather explicitly) from the ones in $\chi_{*}$, modulo a nonlinear transformation involving the pressure law $p(\rho)$. In addition, several properties of "cancellation of singularities" were observed for the function $E\left(\rho, v ; s_{1}, s_{2}\right):=\chi\left(\rho, v ; s_{1}\right) \sigma\left(\rho, v ; s_{2}\right)-\chi\left(\rho, v ; s_{2}\right) \sigma\left(\rho, v ; s_{1}\right)$ and its derivatives of order $\lambda+1$, as $s_{2} \rightarrow s_{1}$.

Then any Young measure satisfying the commutation relation (6.5) for all weak entropy pairs was proved to be a Dirac mass. As in [70], (6.5) can be directly written in terms of the kernels $\chi$ and $\sigma$. The proof relies on the following identity:

$$
\begin{aligned}
& \left\langle\nu_{t, x}, \chi\left(\rho, v ; s_{1}\right)\right\rangle\left\langle\nu_{t, x}, \partial_{s_{2}}^{\lambda+1} \partial_{s_{3}}^{\lambda+1} E\left(\rho, v ; s_{2}, s_{3}\right)\right\rangle \\
& \quad+\left\langle\nu_{t, x}, \partial_{s_{2}}^{\lambda+1} \chi\left(\rho, v ; s_{2}\right)\right\rangle\left\langle\nu_{t, x}, \partial_{s_{3}}^{\lambda+1} E\left(\rho, v ; s_{3}, s_{1}\right)\right\rangle \\
& \quad+\left\langle\nu_{t, x}, \partial_{s_{3}}^{\lambda+1} \chi\left(\rho, v ; s_{3}\right)\right\rangle\left\langle\nu_{t, x}, \partial_{s_{2}}^{\lambda+1} E\left(\rho, v ; s_{1}, s_{2}\right)\right\rangle=0
\end{aligned}
$$


in the sense of distributions for all real variables $s_{j}, j=1,2,3$, where the fractional derivative operator $\partial_{s}^{\lambda+1}$ is used as in $[15,42]$. Then let $s_{2}$ and $s_{3}$ converge to $s_{1}$ and justify that the sum of the second and third terms converge to zero in a weak sense. On the other hand, the first term is highly singular: the main term of interest has the form of the product of a function of bounded variation by a bounded measure, which is not defined in a classical sense and may actually have several rigorous meanings. With this observation, it was shown, with careful analysis, that the first term generally converges weakly to a limit that is not zero. Further delicate analysis is also necessary to show that the other singular terms vanish at the limit. Then the compactness result follows.

With the compactness framework, we can conclude

Theorem 6.5 (Chen-LeFloch [25]). Assume that the initial data $\left(\rho_{0}, m_{0}\right)(x)$ in (6.19) satisfies

$$
0 \leq \rho_{0}(x) \leq C_{0}, \quad\left|m_{0}(x)\right| \leq C_{0} \rho_{0}(x) \quad \text { for a.e. } x \text { and some } C_{0}>0 .
$$

Then

(i) There exists an entropy solution $(\rho, m)$ of the Cauchy problem (6.11) and (6.19), globally defined in time, such that

$$
0 \leq \rho(t, x) \leq C, \quad|m(t, x)| \leq C \rho(t, x) \quad \text { a.e. }(t, x),
$$

where $C$ depends only on $C_{0}$ and the pressure function $p(\cdot)$.

(ii) The solution operator $(\rho, m)(t, \cdot)=S_{t}\left(\rho_{0}, m_{0}\right)(\cdot)$ determined by (i) is compact in $L_{l o c}^{1}\left(\mathbb{R}_{+}^{2}\right)$.

The results in Theorem 6.5 are somewhat surprising, since the flux function of (6.11) is only Lipschitz continuous. The example found by Greenberg-Rascle [57] demonstrates that there exists a system of form (6.10) with only $C^{1}$ (but not $C^{2}$ ) flux function admitting time-periodic and space-periodic solutions, which indicates that the compactness and asymptotic decay of entropy solutions are very sensitive with respect to the smoothness of flux functions.

A compactness framework for the isothermal Euler equation $(\gamma=1)$ has been also established in Huang-Wang [64] (also see LeFloch-Shelukhin [68]). A shock capturing scheme has been developed in Chen-Li [26] to construct shock capturing approximate solutions that converge to an entropy solution in $L^{\infty}$ based on the compactness framework.

By the Euler-Lagrange transformation, the results established here for (6.11) can be reformulated into those for the Lagrangian system (see Wagner [106] and Chen [18]). 
6.4. Two-dimensional sonic-subsonic flows. Consider the two-dimensional Euler equations for steady irrotational flows:

$$
\begin{aligned}
& \partial_{x} v-\partial_{y} u=0, \\
& \partial_{x}(\rho u)+\partial_{y}(\rho v)=0, \\
& \partial_{x}\left(\rho u^{2}+p\right)+\partial_{y}(\rho u v)=0, \\
& \partial_{x}(\rho u v)+\partial_{y}\left(\rho v^{2}+p\right)=0,
\end{aligned}
$$

where $u$ and $v$ are the two components of flow velocity and $\rho$ is the density.

For a polytropic gas with adiabatic exponent $\gamma>1, p=\rho^{\gamma} / \gamma$ is the normalized pressure. Equations (6.26) and (6.28)-(6.29) classically yield Bernoulli's law (cf. $[35])$ :

$$
\rho=\hat{\rho}\left(q^{2}\right):=\left(1-\frac{\gamma-1}{2} q^{2}\right)^{\frac{1}{\gamma-1}},
$$

where $q=\sqrt{u^{2}+v^{2}}$ is the flow speed. The sound speed $c$ is defined as $c^{2}=$ $p^{\prime}(\rho)=1-\frac{\gamma-1}{2} q^{2}$. At the sonic point $q=c, q^{2}=\frac{2}{\gamma+1}$. Then we define the critical speed $q_{c r}:=\sqrt{\frac{2}{\gamma+1}}$ and rewrite Bernoulli's law (6.30) in the form $q^{2}-q_{c r}^{2}=$ $\frac{2}{\gamma+1}\left(q^{2}-c^{2}\right)$. Thus the flow is subsonic when $q<q_{c r}$, sonic when $q=q_{c r}$, and supersonic when $q>q_{c r}$.

For isothermal flow, $p=\bar{c}^{2} \rho$, where $\bar{c}>0$ is the constant sound speed. Then, in the place of (6.30), Bernoulli's law yields

$$
\rho=\hat{\rho}\left(q^{2}\right):=\rho_{0} \exp \left(-\frac{u^{2}+v^{2}}{2 \bar{c}^{2}}\right)
$$

for some constant $\rho_{0}>0$. In this case, $q_{c r}=\bar{c}$.

Let a sequence of functions $\mathbf{w}^{\varepsilon}(x, y)=\left(u^{\varepsilon}, v^{\varepsilon}\right)(x, y)$, defined on an open subset $\Omega \subset \mathbb{R}^{2}$, satisfy the following Set of Conditions (A):

(A.1) $q^{\varepsilon}(x, y)=\left|\mathbf{w}^{\varepsilon}(x, y)\right| \leq q_{c r}$ a.e. in $\Omega$;

(A.2) $\partial_{x} \eta_{k}\left(\mathbf{w}^{\varepsilon}\right)+\partial_{y} q_{k}\left(\mathbf{w}^{\varepsilon}\right), k=1,2,3,4$, are confined in a compact set in $H_{l o c}^{-1}(\Omega)$ for the momentum entropy-entropy flux pairs:

$$
\left(\eta_{1}, q_{1}\right)=\left(\rho u^{2}+p, \rho u v\right), \quad\left(\eta_{2}, q_{2}\right)=\left(\rho u v, \rho v^{2}+p\right),
$$

and the two natural entropy-entropy flux pairs:

$$
\left(\eta_{3}, q_{3}\right)=(v,-u), \quad\left(\eta_{4}, q_{4}\right)=(\rho u, \rho v) .
$$

Then we have 
Theorem 6.6 (Chen-Dafermos-Slemrod-Wang [20]). Let a sequence of functions $\mathbf{w}^{\varepsilon}(x, y)=\left(u^{\varepsilon}, v^{\varepsilon}\right)(x, y)$ satisfy Framework (A). Then the associated Young measure $\nu$ is a Dirac mass and the sequence $\mathbf{w}^{\varepsilon}(x, y)$ is compact in $L^{1}(\Omega)$; that is, there is a subsequence (still labeled) $\mathbf{w}^{\varepsilon}$ that converges a.e. as $\varepsilon \rightarrow 0$ to $\mathbf{w}=(u, v)$ satisfying

$$
q(x, y)=|\mathbf{w}(x, y)| \leq q_{c r} \quad \text { a.e. }(x, y) \in \Omega .
$$

The proof is based on the compensated compactness argument to reduce the Young measure in the commutation relation as $(6.5)$, with $(x, y)$ replacing $(t, x)$, by using only the two momentum entropy pairs in (6.32), besides the two natural entropy pairs in (6.33).

We now consider a sequence of approximate solutions $\mathbf{w}^{\varepsilon}(x, y)$ to the Euler equations (6.26)-(6.27) with Bernoulli's law (6.30) or (6.31). That is, besides the Set of Conditions $(\mathrm{A})$, the sequence $\mathbf{w}^{\varepsilon}(x, y)=\left(u^{\varepsilon}, v^{\varepsilon}\right)(x, y)$ further satisfies

$$
\begin{aligned}
& \partial_{x} v^{\varepsilon}-\partial_{y} u^{\varepsilon}=o_{1}^{\varepsilon}(1), \\
& \partial_{x}\left(\hat{\rho}\left(\left|\mathbf{w}^{\varepsilon}\right|^{2}\right) u^{\varepsilon}\right)+\partial_{y}\left(\hat{\rho}\left(\left|\mathbf{w}^{\varepsilon}\right|^{2}\right) v^{\varepsilon}\right)=o_{2}^{\varepsilon}(1),
\end{aligned}
$$

where $o_{j}^{\varepsilon}(1) \rightarrow 0, j=1,2$, in the sense of distributions as $\varepsilon \rightarrow 0$. Then, as a corollary of the compensated compactness framework (Theorem 6.6), we conclude that there exists a subsequence (still labeled) $\mathbf{w}^{\varepsilon}(x, y)$ that converges a.e. as $\varepsilon \rightarrow 0$ to a weak solution $\mathbf{w}=(u, v)$ of the Euler equations $(6.26)-(6.27)$ with Bernoulli's law (6.30) or (6.31) satisfying

$$
q(x, y)=|\mathbf{w}(x, y)| \leq q_{c r} \quad \text { a.e. }(x, y) \in \Omega .
$$

Even though the flow may eventually turn out to be smooth, the point of considering here weak solutions is to demonstrate that such solutions may be constructed by merely using very crude estimates, which are readily available in a variety of approximating methods.

We now consider the sonic limit of subsonic flows past an obstacle $\mathcal{P}$, such as an airfoil. As in Bers [7], write

$$
\mathbf{z}=x+i y, \quad \mathbf{w}=u-i v=q e^{-i \theta}, \quad q=\sqrt{u^{2}+v^{2}} ; \quad u=q \cos \theta, \quad v=-q \sin \theta .
$$

Consider a fixed simple closed rectifiable curve $\mathcal{C}$ (the boundary of the obstacle $\mathcal{P}$ ) in the z-plane and a fixed point $\mathbf{z}_{T}$ on it (the trailing edge). This curve may possess at $\mathbf{z}_{T}$ a protruding corner or cusp, but should otherwise be a Lyapunov curve (a Lyapunov curve is a curve that possesses a tangent which satisfies a Hölder condition with respect to the arc length). Let $S$ be the length of $\mathcal{C}$, and $\delta \pi$ the opening of the corner at $\mathbf{z}_{T}$. If $\delta=0, \mathcal{C}$ has a cusp at $\mathbf{z}_{T}$; if $\delta=1$, $\mathcal{C}$ possesses tangent at $\mathbf{z}_{T}$; otherwise, $0<\delta<1$. The profile $\mathcal{C}$ admits the 
parametric representation:

$$
\mathbf{z}=\mathbf{z}_{T}+\int_{0}^{s} e^{i \Theta(\sigma)} d \sigma, \quad 0 \leq s \leq S .
$$

The function $\Theta(s)$ must satisfy the condition

$$
\Theta(S)-\Theta(0)=(1+\delta) \pi, \quad 0 \leq \delta \leq 1,
$$

and the Hölder condition

$$
\left|\Theta\left(s_{2}\right)-\Theta\left(s_{1}\right)\right| \leq k\left(s_{2}-s_{1}\right)^{\alpha}, \quad 0 \leq s_{1}<s_{2} \leq S,
$$

for some constants $k>0$ and $0<\alpha<1$.

Denote by $\mathbb{D}(\mathcal{C})$ the domain exterior to $\mathcal{C}$. A pair of functions $(u, v) \in C^{1}(\mathbb{D}(\mathcal{C}))$ is called a solution of Problem $\mathcal{P}$, if $(u, v)$ satisfies (6.26)-(6.27) with Bernoulli's law (6.30) or (6.31), and the slip boundary condition

$$
(u, v) \cdot \nu=0 \quad \text { on } \quad \mathcal{C},
$$

where $\nu$ denotes the normal on $\mathcal{C}$, and the limit $\mathbf{w}_{\infty}:=\lim _{\mathbf{z} \rightarrow \infty}(u-i v)$ exists and is finite.

A pair of functions $(u, v)$ defined on $\mathbb{D}(\mathcal{C})$ is said to satisfy the Kutta-Joukowski condition if

$$
u^{2}+v^{2} \rightarrow 0 \quad \text { as } \quad \mathbf{z} \rightarrow \mathbf{z}_{T}, \quad \text { if } \quad \delta=1
$$

(a stagnation point at the trailing edge), or

$$
u^{2}+v^{2}=O(1) \quad \text { as } \quad \mathbf{z} \rightarrow \mathbf{z}_{T}, \quad \text { if } \quad 0 \leq \delta<1 .
$$

Any solution of Problem $\mathcal{P}$ automatically satisfies the Kutta-Joukowski condition if $0 \leq \delta<1$; in particular, for such a function,

$$
u^{2}+v^{2}=0 \quad \text { at } \quad \mathbf{z}_{T}, \quad \text { if } \quad 0<\varepsilon<1 .
$$

Also, with every solution of Problem $\mathcal{P}$, we associate the circulation $\Gamma$ as

$$
\Gamma=\oint_{\mathcal{C}}(u, v) \cdot \mathbf{t} d s,
$$

where $\mathbf{t}$ is the unit tangent to $\mathcal{C}$.

Bers [7] considered the following two boundary value problems:

Problem $\mathcal{P}_{1}\left(\mathbf{w}_{\infty}\right): \quad$ Find a solution of Problem $\mathcal{P}$ satisfying a Kutta-Joukowski condition and a prescribed limit $\mathbf{w}_{\infty}$ as $\mathbf{z} \rightarrow \infty$.

Problem $\mathcal{P}_{2}\left(\mathbf{w}_{\infty}, \Gamma\right): \quad$ Find a solution of Problem $\mathcal{P}$ for which $\mathbf{w}_{\infty}$ at $\mathbf{z}=\infty$ and $\Gamma$ on the boundary $\mathcal{C}$ are prescribed.

Problem $\mathcal{P}_{2}$ is only considered in the case of a smooth profile $\delta=1$. 
Theorem 6.7 (Bers [7]). For a given $\mathbf{w}_{\infty}$, there exists a number $\hat{q}<q_{c r}$, depending on the profile and the equation of state, such that Problem $\mathcal{P}_{1}\left(\mathbf{w}_{\infty}\right)$ has a unique solution $(u, v)$ for $q_{\infty}:=\left|\mathbf{w}_{\infty}\right|<\hat{q}$. The velocity $(u, v)$ is Hölder continuous on the profile and depends continuously on $\mathbf{w}_{\infty}$. The maximum speed $q_{m}$ of $|\mathbf{w}|$ takes on all values between 0 and some critical value $q_{c r}$, and $q_{m} \rightarrow 0$ as $q_{\infty} \rightarrow 0, q_{m} \rightarrow q_{c r}$ as $q_{\infty} \rightarrow \hat{q}$. A similar result holds for Problem $\mathcal{P}_{2}\left(\mathbf{w}_{\infty}, \Gamma\right)$.

The proof of the Bers's existence-uniqueness theorem simplifies an earlier proof of Shiffman [95] on the existence of solutions. Bers's Theorem does not apply to the critical flows, that is, those flows for which $q_{\infty}=\hat{q}$ and which hence must be sonic at some point in $\mathbb{D}(\mathcal{C}) \cup \mathcal{C}$. In fact, the Gilbarg-Shiffman maximum principle [53] asserts that the sonic point should occur on $\mathcal{C}$, which presupposed the existence of critical flows. The following more general result has been established.

Theorem 6.8 (Chen-Dafermos-Slemrod-Wang [20]). Let $q^{\varepsilon}<\hat{q}$ be a sequence of speeds at $\infty$, and let $\left(u^{\varepsilon}, v^{\varepsilon}\right)$ be the corresponding solutions to either Problem $\mathcal{P}_{1}\left(\mathbf{w}_{\infty}\right)$ or $\mathcal{P}_{2}\left(\mathbf{w}_{\infty}, \Gamma\right)$. Then, as $q^{\varepsilon} \nearrow \hat{q}$, the solution sequence $\left(u^{\varepsilon}, v^{\varepsilon}\right)$ possesses a subsequence (still denoted by) $\left(u^{\varepsilon}, v^{\varepsilon}\right)$ that converges strongly a.e. in $\mathbb{D}(\mathcal{C})$ to a pair of functions $(u, v)$ which is a weak solution of equations (6.26)-(6.27) with Bernoulli's law (6.30) or (6.31). Furthermore, the limit velocity $(u, v)$ satisfies the boundary conditions (6.38) as the normal trace of the divergence-measure field $(\rho u, \rho v)$ on the boundary (cf. [22]).

The strong solutions $\mathbf{w}^{\varepsilon}:=\left(u^{\varepsilon}, v^{\varepsilon}\right)$ in Theorem 6.7 satisfy (6.28)-(6.29) and are subsonic so that the sequenca $\mathbf{w}^{\varepsilon}(x, y)$ satisfies the compactness framework, which implies that the Young measures are Dirac masses and the convergence is strong a.e. in $\mathbb{D}(\mathcal{C})$. The fact that the boundary conditions (6.38) are satisfied for $(u, v)$ in the sense of distributions is standard by multiplying (6.27) by a test function and applying the Gauss-Green Theorem and by the fact that the sequence of subsonic solutions does satisfy $(6.38)$, which implies that $(u, v)$ satisfies the boundary conditions (6.38) actually as the normal trace of the divergencemeasure field $(\rho u, \rho v)$ on the boundary in Theorem 2.1 (cf. Chen-Frid [22]).

\section{Further REMARKS AND OUTLOOK}

Some new measure-theoretic methods have been also developed in Chen-Perthame [29] and Chen-Karlsen [24] for kinetic and entropy solutions, Perthame-Souganidis [86] for dissipative and entropy solutions, and Tadmor-Tao [101] for velocity averaging and regularizing effects to handle nonlinear anisotropic degenerate parabolic-hyperbolic equations.

Many fundamental problems in the theory of conservation laws for bounded entropy solutions, but without bounded variation, are open and challenging. This is 
particularly so for systems of conservation laws, and even for the one-dimensional case. These include the traces, generalized characteristics, uniqueness and stability, and asymptotic behavior of entropy solutions only in $L^{\infty}$. The multidimensional problems are even richer and much more complicated and will require new techniques and approaches in which measure-theoretic analysis will play an important role.

Acknowledgments. Gui-Qiang Chen's research was supported in part by the National Science Foundation under Grants DMS-0505473, DMS-0244473, and an Alexander von Humboldt Foundation Fellowship. Monica Torres's research was supported in part by the National Science Foundation under grant DMS-0540869.

\section{REFERENCES}

[1] Ambrosio, L., Crippa, G., and Maniglia, S., Traces and fine properties of a BD class of vector fields and applications, Ann. Fac. Sci. Toulouse Math. (6), 14 (4) (2005), 527-561.

[2] Ambrosio, L., Fusco, N., and Pallara, D., Functions of Bounded Variation and Free Discontinuity Problems, The Clarendon Press, Oxford University Press: New York, 2000.

[3] Ambrosio, L. and De Lellis, C., Existence of solutions for a class of hyperbolic systems laws in several space dimensions, Int. Math. Res. Not. 41 (2003), 2205-2220.

[4] Anzelloti, G., Pairings between measures and functions and compensated compactness, Anal. Mat. Pure Appl. 135 (1983), 293-318.

[5] Baiocchi, C. and Capelo, A., Variational and Quasi-variational Inequalities: Applications to Free-Boundary Problems, Vols. 1,2, Wiley: Chichester-New York, 1984.

[6] Ball, J., A version of the fundamental theorem for Young measures, In: Lecture Notes in Physics, 344, Springer-Verlag, 1989, pp 207-215.

[7] Bers, L., Results and conjectures in the mathematical theory of subsonic and transonic gas flows, Comm. Pure Appl. Math. 7 (1954), 79-104; Existence and uniqueness of a subsonic flow past a given profile, Comm. Pure Appl. Math. 7 (1954), 441-504; Mathematical Aspects of Subsonic and Transonic Gas Dynamics, John Wiley \& Sons, Inc.: New York; Chapman \& Hall, Ltd.: London 1958.

[8] Bianchini, S. and Bressan, A., Vanishing viscosity solutions of nonlinear hyperbolic systems, Ann. Math. (2), 161 (2005), 223-342.

[9] Brenner, P., The Cauchy problem for the symmetric hyperbolic systems in $L_{p}$, Math. Scand. 19 (1966), 27-37.

[10] Bressan, A., Hyperbolic Systems of Conservation Laws: The One-dimensional Cauchy Problem, Oxford University Press: Oxford, 2000.

[11] Bressan, A., An ill posed Cauchy problem for a hyperbolic system in two space dimensions, Rend. Sem. Mat. Univ. Padova, 110 (2003), 103-117.

[12] Brezzi, F. and Forti, M., Mixed and Hybrid Finite Element Method, Springer-Verlag: New York, 1991.

[13] Burago, Yu. D. and Maźya, V. G., Potential Theory and Function Theory for Irregular Regions, Translated fron Russian, Seminars in Math., V. A. Steklov Mathematical Institute, Leningrad, Vol. 3, Consultants Bureau: New York, 1969. 
[14] Cauchy, A.-L., Recherches sur l'équilibre et le mouvement intérieur des corps solides ou fluides, élastiques or non élastiques, Bull. Soc. Philomathique (1823), 9-13; Da la pression ou tension dans un corps solide, Exercises de Matehématiques, 2 (1827), 42-56.

[15] Chen, G.-Q., Convergence of the Lax-Friedrichs scheme for isentropic gas dynamics (III), Acta Math. Sci. 6 (1986), 75-120 (in English); 8 (1988), 243-276 (in Chinese).

[16] Chen, G.-Q., The compensated compactness method and the system of isentropic gas dynamics, MSRI Preprint 00527-91, 1990.

[17] Chen, G.-Q., Hyperbolic systems of conservation laws with a symmetry, Commun. Partial Diff. Eqs. 16 (1991), 1461-1487.

[18] Chen, G.-Q., The method of quasidecoupling for discontinuous solutions to conservation laws, Arch. Rational Mech. Anal. 121 (1992), 131-185.

[19] Chen, G.-Q., Compactness Methods and Nonlinear Hyperbolic Conservation Laws, AMS/IP Stud. Adv. Math. 15, 33-75, AMS: Providence, RI, 2000; Some recent methods for partial differential equations of divergence form, Bull. Braz. Math. Soc. (N.S.), 34 (2003), 107-144; Euler Equations and Related Hyperbolic Conservation Laws, In: Handbook of Differential Equations, Vol. 2, Eds. C. M. Dafermos and E. Feireisl, pp. 1-104, Elsevier Science B.V: Amsterdam, The Netherlands, 2005.

[20] Chen, G.-Q., Dafermos, C. M., Slemrod, M., and Wang, D., On two-dimensional sonicsubsonic flow, Commun. Math. Phys. 271 (2007), 635-647.

[21] Chen, G.-Q. and Feldman, M., Multidimensional transonic shocks and free boundary problems for nonlinear equations of mixed type, J. Amer. Math. Soc. 16 (2003), 461-494.

[22] Chen, G.-Q. and Frid, H., Divergence-measure fields and hyperbolic conservation laws, Arch. Rational Mech. Anal. 147 (1999), 89-118; Extended divergence-measure fields and the Euler equations of gas dynamics, Commun. Math. Phys. 236 (2003), 251-280.

[23] Chen, G.-Q. and Frid, H., Decay of entropy solutions of nonlinear conservation laws, Arch. Rational Mech. Anal. 146 (1999), 95-127; Large-time behavior of entropy solutions for multidimensional conservation laws, In: Advances in Nonlinear Partial Differential Equations and Related Areas, pp. 28-44, World Sci.: Singapore, 1998; Large-time behavior of entropy solutions of conservation laws, J. Diff. Eqs. 152 (1999), 308-357.

[24] Chen, G.-Q. and Karlsen, K., Quasilinear anisotropic degenerate parabolic equations with time-space dependent diffusion coefficients, Comm. Pure Appl. Anal. 4 (2005), 241-266; Continuous dependence and error estimates for quasilinear degenerate parabolic equations: Kinetic approach, Trans. Amer. Math. Soc. 358 (2006), 937-963.

[25] Chen, G.-Q. and LeFloch, Ph., Compressible Euler equations with general pressure law, Arch. Rational Mech. Anal. 153 (2000), 221-259; Existence theory for the isentropic Euler equations, Arch. Rational Mech. Anal. 166 (2003), 81-98.

[26] Chen, G.-Q. and Li, T.-H., Global entropy solutions in $L^{\infty}$ to the Euler equations and Euler-Poisson equations for isothermal fluids with spherical symmetry, Meth. Appl. Anal. 10 (2003), 215-244.

[27] Chen, G.-Q., Li, B.-H., and Li, T.-H., Entropy solutions in $L^{\infty}$ for the Euler equations in nonlinear elastodynamics and related equations, Arch. Rational Mech. Anal. 170 (2003), 331-357.

[28] Chen, G.-Q. and Lu, Y., A study of approaches to applying the theory of compensated compactness, Chinese Sci. Bull. 34 (1989), 15-19 (in English); 33 (1988), 641-644 (in Chinese).

[29] Chen, G.-Q. and Perthame, B., Well-posedness for anisotropic degenerate parabolichyperbolic equations, Ann. l'Institut Henri Poincaré: Analyse Non Linéaire, 20 (2003), 645-668; Decay of periodic solutions to anisotropic degenerate parabolic-hyperbolic equations, Preprint, 2006. 
[30] Chen, G.-Q. and Rascle, M., Initial layers and uniqueness of weak entropy solutions to hyperbolic conservation laws, Arch. Rational Mech. Anal. 153 (2000), 205-220.

[31] Chen, G.-Q. and Torres, M., Divergence measure fields, sets of finite perimeter, and conservation laws, Arch. Rational Mech. Anal. 175 (2005), 245-267.

[32] Chen, G.-Q., Torres, M. and Ziemer, W., Gauss-Green theorem for weakly differentiable vector fields, sets of finite perimeter, and balance laws, Preprint, March 2006.

[33] Chen, G.-Q. and Wang, D., The Cauchy Problem for the Euler Equations for Compressible Fluids, Handbook of Mathematical Fluid Dynamics, Vol. 1, pp. 421-543, Elsevier Science B. V: Amsterdam, The Netherlands, 2002.

[34] Coifman, R. R., Lions, P.-L., Meyer, Y., and S. Semmes, Compensated compactness and Hardy spaces, J. Math. Pures Appl. (9), 72 (1993), 247-286.

[35] Courant, R. and Friedrichs, K. O., Supersonic Flow and Shock Waves, Springer-Verlag: New York, 1962.

[36] Dacorogna, B., Weak Continuity and Weak Lower Semicontinuity of Nonlinear Functionals, Lecture Notes in Math. 922, Springer-Verlag: Berlin-New York, 1982; Direct Methods in the Calculus of Variations, Springer-Verlag: Berlin-New York, 1989.

[37] Dafermos, C. M., Hyperbolic Conservation Laws in Continuum Physics, Springer-Verlag: New York, 1999.

[38] Dafermos, C. M., Large time behavior of periodic solutions of hyperbolic systems of conservation laws, J. Diff. Eqs. 121 (1995), 183-202.

[39] De Lellis, C., Blowup of the BV norm in the multidimensional Keyfitz and Kranzer system, Duke Math. J. 127 (2005), 313-339.

[40] De Lellis, C. and Otto, F., and Westdickenberg, M., Structure of entropy solutions for multidimensional scalar conservation laws, Arch. Rational Mech. Anal. 170 (2003), 137184.

[41] Ding, X., Zhang, T., Wang, C.-H., Hsiao, L., and Li, T.-C., A study of the global solutions for quasilinear hyperbolic systems of conservation laws, Scientica Sinica, 16 (1973), 317335 .

[42] Ding, X., Chen, G.-Q., and Luo, P., Convergence of the Lax-Friedrichs scheme for isentropic gas dynamics (I)-(II), Acta Math. Sci. 5 (1985), 483-500, 501-540 (in English); 7 (1987), 467-480, 8 (1988), 61-94 (in Chinese).

[43] DiPerna, R. J., Convergence of viscosity method for isentropic gas dynamics, Commun. Math. Phys. 91 (1983), 1-30.

[44] DiPerna, R. J., Convergence of approximate solutions to conservation laws, Arch. Rational Mech. Anal. 82 (1983), 27-70.

[45] DiPerna, R.-J., Lions, P. -L, and Meyer, Y., $L^{p}$ regularity of velocity averages, Ann. Inst. H. Poincaré Anal. Non Linéaire, 8 (1991), 271-287.

[46] Evans, L. C., Weak Convergence Methods for Nonlinear Partial Differential Equations, CBMS Regional Conf. Series in Math. AMS: Providence, 1990.

[47] Evans, L. C. and Gariepy, R. F., Measure Theory and Fine Properties of Functions, CRC Press: Boca Raton, FL, 1992.

[48] Federer, H., Geometric Measure Theory, Springer-Verlag New York Inc.: New York, 1969

[49] Finn, R., On the flow of a perfect fluid through a polygonal nozzle, I, II, Proc. Nat. Acad. Sci. USA. 40 (1954), 983-985, 985-987; On a problem of type, with application to elliptic partial differential equations, J. Rational Mech. Anal. 3 (1954), 789-799.

[50] Finn, R. and Gilbarg, D., Asymptotic behavior and uniquenes of plane subsonic flows, Comm. Pure Appl. Math. 10 (1957), 23-63; Three-dimensional subsonic flows, and asymptotic estimates for elliptic partial differential equations, Acta Math. 98 (1957), 265296; Uniqueness and the force formulas for plane subsonic flows, Trans. Amer. Math. Soc. 88 (1958), 375-379. 
[51] Friedrichs, K. O. and Lax, P. D., Systems of conservation equations with a convex extension, Proc. Nat. Acad. Sci. U.S.A. 68 (1971), 1686-1688.

[52] Gilbarg, D. and Serrin, J., Uniqueness of axially symmetric subsonic flow past a finite body, J. Rational Mech. Anal. 4 (1955), 169-175.

[53] D. Gilbarg and M. Shiffman, On bodies achieving extreme values of the critical Mach number, I, J. Rational Mech. Anal. 3 (1954), 209-230.

[54] Glimm, J., Solutions in the large for nonlinear hyperbolic systems of equations, Comm. Pure Appl. Math. 18 (1965), 95-105.

[55] Glimm, J. and Lax, P. D., Decay of Solutions of Systems of Hyperbolic Conservation Laws, Mem. Amer. Math. Soc. 101, AMS: Providence, 1970.

[56] Golse, F., Lions, P.-L., Perthame, B., and Sentis, R., Regularity of the moments of the solution of a transport equation, J. Funct. Anal. 76 (1988), 110-125.

[57] Greenberg, J. M. and Rascle, M., Time-periodic solutions to systems of conservation laws, Arch. Rational Mech. Anal. 115 (1991), 395-407.

[58] Gripenberg, G., Compensated compactness and one-dimensional elastodynamics, Ann. Scuola Norm. Sup. Pisa Cl Sci. (4), 22 (1995), 227-240.

[59] Gurtin, M. E. and Martins, L. C., Cauchy's theorem in classical physics, Arch. Rational Mech. Anal. 60 (1976), 305-324.

[60] Gurtin, M. E. and Williams, W. O., An axiomatic foundation for continuum thermodynamics, Arch. Rational Mech. Anal. 26 (1967), 83-117.

[61] Hardt, R. and Simon, L., Seminar on Geometric Measure Theory, DMV Seminar, 7, Birkhäuser Verlag: Basel, 1986.

[62] Holden, H. and Risebro, N. H., Front Tracking for Hyperbolic Conservation Laws, Springer-Verlag: New York, 2002.

[63] Hörmander, L., Lectures on Nonlinear Hyperbolic Differential Equations, Springer-Verlag: Berlin, 1997.

[64] Huang, F.-M. and Wang, Z., Convergence of viscosity solutions for isothermal gas dynamics, SIAM J. Math. Anal. 34 (2002), 595-610.

[65] Kwon, Y.-S. and Vasseur, A., Strong traces for solutions of scalar conservation laws with general flux, Arch. Rational Mech. Anal. 2007 (in press).

[66] Lax, P. D., Hyperbolic Systems of Conservation Laws and the Mathematical Theory of Shock Waves, SIAM: Philadelphia, 1973.

[67] LeFloch, Ph., Hyperbolic Systems of Conservation Laws: The Theory of Classical and Nonclassical Shock Waves, Lectures in Mathematics ETH Zürich, Birkhäuser Verlag: Basel, 2002.

[68] LeFloch, Ph. and Shelukhin, V., Symmetries and global solvability of the isothermal gas dynamics equations, Arch. Rational Mech. Anal. 175 (2005), 389-430.

[69] Lin, F.-H. and Yang, X.-P., Geometric Measure Theory-An Introduction, Advanced Mathematics (Beijing/Boston), 1, Science Press: Beijing; International Press: Boston, MA, 2002.

[70] Lions, P.-L., Perthame, B., and Souganidis, P., Existence and stability of entropy solutions for the hyperbolic systems of isentropic gas dynamics in Eulerian and Lagrangian coordinates, Comm. Pure Appl. Math. 49 (1996), 599-638.

[71] Lions, P.-L., Perthame, B., and Tadmor, E., Kinetic formulation of the isentropic gas dynamics and p-systems, Commun. Math. Phys. 163 (1994), 169-172.

[72] Lions, P. L. and Perthame, B. and Tadmor E., A kinetic formulation of multidimensional scalar conservation laws, J. Amer. Math. Soc. 7 (1994), 169-191.

[73] Liu, T.-P., The deterministic version of the Glimm scheme, Commun. Math. Phys. 57 (1977), 135-148. 
[74] Maly, J. and Ziemer, W. P., Fine Regularity of Solutions of Elliptic Partial Differential Equations, Mathematical Surveys and Monographs, Vol. 51, AMS: Providence, 1997.

[75] McShane, E. J., Integration, Princeton University Press: Princeton, 1944.

[76] Morawetz, C. S., The mathematical approach to the sonic barrier, Bull. Amer. Math. Soc. (N.S.) 6 (1982), 127-145; On a weak solution for a transonic flow problem, Comm. Pure Appl. Math. 38 (1985), 797-818; On steady transonic flow by compensated compactness, Methods Appl. Anal. 2 (1995), 257-268; Mixed equations and transonic flow, J. Hyper. Diff. Eqs. 1 (2004), 1-26.

[77] Morry, C. B., Quasiconvexity and the lower semicontinuity of multiple integrals, Pacific J. Math. 2 (1952), 25-53; Multiple Integrals in the Calculus of Variations, Springer-Verlag: Berlin-New York, 1966.

[78] Murat, F., Compacite par compensation, Ann. Suola Norm. Pisa (4), 5 (1978), 489-507.

[79] Murat, F., L'injection du cone positif de $H^{-1}$ dans $W^{-1, q}$ est compacte pour tout $q<2$, J. Math. Pure Appl. 60 (1981), 309-322.

[80] Murat, F., Compacite par compensation: condition necessarie et suffisante de continuite faible sous une hypothese de range constant, Ann. Scuola Norm. Sup. Pisa (4), 8 (1981), 69-102; A survey on compensated compactness, In: Contributions to Modern Calculus of Variations (Bologna, 1985), 145-183, Pitman Res. Notes Math. Ser. 148, Longman Sci. Tech.: Harlow, 1987.

[81] Nishida, T., Global solution for an initial-boundary value problem of a quasilinear hyperbolic systems, Proc. Japan Acad. 44 (1968), 642-646.

[82] T. Nishida and J. Smoller, Solutions in the large for some nonlinear hyperbolic conservation laws, Comm. Pure Appl. Math. 26 (1973), 183-200.

[83] Noll, W., The foundations of classical mechanics in the light of recent advances in continuum mechanics, In: The Axiomatic Method, pp. 266-281, Amsterdam: North Holland, 1959.

[84] Panov, E. Yu., Existence of strong traces for generalized solutions of multidimensional scalar conservation laws, J. Hyper. Diff. Eqns. 2 (2005), 885-908.

[85] Perthame, B. and Souganidis, P., A limiting case for velocity averaging, Ann. Sci. École Norm. Sup. (4), 31 (1998), 591-598.

[86] Perthame, B. and Souganidis, P., Dissipative and entropy solutions to non-isotropic degenerate parabolic balance laws, Arch. Rational Mech. Anal. 170 (2003), 359-370; Addendum to: "Dissipative and entropy solutions to non-isotropic degenerate parabolic balance laws", Arch. Rational Mech. Anal. 174 (2004), 443-447.

[87] Perthame, B. and Tzavaras, A. E., Kinetic formulation for systems of two conservation laws and elastodynamics, Arch. Rational Mech. Anal. 155 (2000), 1-48.

[88] Rauch, J., BV estimates fail for most quasilinear hyperbolic systems in dimensions greater than one, Commun. Math. Phys. 106 (1986), 481-484.

[89] B. Riemann, Uber die Fortpflanzung ebener Luftwellen von endlicher Schwingungsweite, Abhandl. Koenig. Gesell. Wiss., Goettingen, 8 (1860), 43-65.

[90] Rodriguez, J.-F., Obstacle problems in Mathematical Physics, North-Holland Mathematics Studies, 134, Elsevier Science Publishers B.V., 1987.

[91] Schoen, R. and Yau, S.-T., Lectures on Differential Geometry, International Press: Cambridge, MA, 1994.

[92] Schonbek, M. E., Convergence of solutions to nonlinear dispersive equations, Commun. Partial Diff. Eqs. 7 (1982), 959-1000.

[93] Serre, D., La compacité par compensation pour les systèmes hyperboliques non linéaires de deux équations à une dimension d'espace (French), J. Math. Pures Appl. (9), 65 (1986), 423-468. 
[94] Serre, D., Systems of Conservation Laws, Vols. 1-2, Cambridge University Press: Cambridge, 1999, 2000.

[95] Shiffman, M., On the existence of subsonic flows of a compressible fluid, J. Rational Mech. Anal. 1 (1952), 605-652.

[96] Simon, L., Lectures on Geometric Measure Theory, Proceedings of the Centre for Mathematical Analysis, Australian National University, 3, Australian National University, Centre for Mathematical Analysis, Canberra, 1983.

[97] Simon, L., The minimal surface equation, Geometry, V, pp. 239-272, Encyclopaedia Math. Sci. 90, Springer: Berlin, 1997.

[98] Simon, L., Theorems on Regularity and Singularity of Energy Minimizing Maps, Lectures in Mathematics, ETH Zürich. Birkhäuser Verla: Basel, 1996.

[99] Stein, E., Singular Integrals and Differentiability Properties of Functions, Princeton University Press: Princeton, New Jersey, 1970.

[100] Struwe, M., Variational Methods: Applications to Nonlinerar Partial Differential Equations and Hamiltonian Systems, Springer-Verlag: Berlin, 1990.

[101] Tadmor, E. and Tao, T., Velocity averaging, kinetic formulations and regularizing effects in quasilinear PDEs, Comm. Pure Appl. Math. 2007 (in press).

[102] Tartar, L., Compensated compactness and applications to partial differential equations, In: Research Notes in Mathematics, Nonlinear Analysis and Mechanics, 4 (ed. R. J. Knops), Pitman Press: New York, 1979.

[103] Tartar, L., Une nouvelle methode de resolution d'equations aux derivees partielles nonlineaires, Lecture Notes in Mathematics, 665, 228-241, Springer-Verlag, 1977; The compensated compactness method applied to systems of conservation laws, In: Systems of Nonlinear PDEs., J. M. Ball (eds.), pp. 263-285, NATO series, C. Reidel publishing Co. 1983; Discontinuities and oscillations, In: Directions in Partial Differential Equations (Madison, WI, 1985), 211-233, Academic Press: Boston, 1987; $H$-measures, a new approaches for studying homogenisation, oscillations and concentration effects in partial differential equations, Proc. Roy. Soc. Edinburgh, 115A (1990), 193-230.

[104] Vasseur, A., Strong traces for solutions to multidimensional scalar conservation laws, Arch. Rational Mech. Anal. 160 (2001), 181-193.

[105] Volpert, A. I. and Hudjaev, S. I., Analysis in Classes of Discontinuous Functions and Equations of Mathematical Physics, Martinus Nijhoff Publishers: Dordrecht, 1985.

[106] Wagner, D. H., Equivalence of the Euler and Lagrangian equations of gas dynamics for weak solutions, J. Diff. Eqs. 68 (1987), 118-136.

[107] Whitney, H., Geometric Integration Theory, Princeton University Press: Princeton, 1957.

[108] Willard, S., General Topology, Addison-Wesley Publishing Co.: Reading, Mass.-LondonDon Mills, Ont., 1970.

[109] Young, L. C., Generalized curves and existence of an attained absolute minimum in the calculus of variations, C. R. Soc. Sci. Lett. Varsovie, Classe III, 30 (1937), 212-234; Generalized surfaces in the calculus of variations (I)-(II), Ann. Math. 43 (1942), 84-103, 530-544; Lectures on the Calculus of Variations and Optimal Control Theory, W. B. Saunders: Philadelphia, 1969.

[110] Zhang, T. and Guo, Y.-F., A class of initial-value problem for systems of aerodynamic equations, Acta Math. Sinica, 15 (1965), 386-396.

[111] Ziemer, W. P., Cauchy flux and sets of finite perimeter, Arch. Rational Mech. Anal. 84, 189-201 (1983).

[112] Ziemer, W. P., Weakly Differentiable Functions: Sobolev Spaces and Functions of Bounded Variation, Springer-Verlag: New York, 1989. 
G.-Q. Chen

Department of Mathematics, Northwestern University 2033 Sheridan Road, Evanston, IL 60208-2730, USA.

http://www.math.northwestern.edu/ ${ }^{\sim}$ gqchen

E-mail: gqchen@math.northwestern.edu

M. Torres

Department of Mathematics

Purdue University, 150 N. University Street

West Lafayette, IN 47907-2067, USA.

E-mail: torres@math.purdue.edu

W. Ziemer

Department of Mathematics, Indiana University

Rawles Hall, Bloomington, IN 47405, USA.

http://www.indiand.edu/ ziemer

E-mail: ziemer@indiana.edu 